\title{
Shedding light on new Wess-Zumino couplings with their corrections to all orders in alpha-prime
}

\author{
Ehsan Hatefi \\ International Centre for Theoretical Physics, \\ Strada Costiera 11, Trieste, Italy \\ Theory Group, Physics Department, CERN, \\ CH-1211, Geneva 23, Switzerland \\ E-mail: ehatefi@ictp.it
}

ABSTRACT: Motivated by arXiv:1203.5553, we continue to match super string amplitudes with their own effective field theory. We carry out within full details the computations of the complete form of the amplitude of one closed string Ramond-Ramond field and three SYM vertex operators, namely one gauge field and two scalar fields in type IIB(A) super string theories. Making use of the recent two gauge and two scalar couplings to all orders of $\alpha^{\prime}$, we produce all the infinite gauge poles for $p=n$ case (with $n$ as RR field strength's rank and $p$ as the dimension of a $\mathrm{D}_{p}$-brane). Proceeding with direct and full S-Matrix calculations, we are able to produce even all the infinite gauge poles in $\mathrm{u}$-channel for $p-2=n$ case in field theory as well. New couplings for $p-2=n$ case with their all order $\alpha^{\prime}$ corrections are discovered. In addition, we explain how to find out all the infinite scalar poles of this amplitude in $s, t$-channels and produce them for $p+2=n$ case. By comparing all of the contact terms of this amplitude, we obtain several new couplings with their higher derivative corrections for $p+2=n, p=n$ cases. These new couplings are neither inside Myers'terms nor within pull-back/Taylor expansions. Finally we comment on some related issues.

Keywords: p-branes, Superstrings and Heterotic Strings, Chern-Simons Theories, String Field Theory

ArXiv EPRINT: 1211.2413 


\section{Contents}

1 Introduction 1

2 Complete form of the $C A \phi \phi$ amplitude to all orders of $\alpha^{\prime} \quad 4$

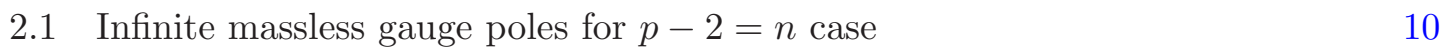

3 New couplings for BPS-branes for $n=p-2$ case 11

$4 \quad$ Infinite massless gauge poles for $p=n$ case $\quad 12$

4.1 Infinite massless $t, s$-channel scalar poles for $p+2=n$ case $\quad 16$

5 Contact interactions for $p=n$ case $\quad 19$

6 New couplings for $p=n$ case $\quad 21$

7 Contact terms for $p+2=n$ case $\quad 23$

$\begin{array}{llr}8 & \text { Conclusion } & 27\end{array}$

\section{Introduction}

$\mathrm{D}_{p}$-branes have been centering in String theory on both theoretical and phenomenological approaches for a while. For diverse values of $p$ (where $p$ is spatial dimension of a $\mathrm{D}_{p}$-brane) and also in both type IIA and IIB string theories, they have been known as the sources of closed string Ramond-Ramond field [1-4].

By computing some of the couplings of $\mathrm{D}_{p}$-branes to closed string modes, some great information have been obtained. We address various examples such as the Ads/CFT correspondence, gauge theory and black holes. Concerning Ramond-Ramond couplings [5, $6]$, diverse phenomena such as $[7,8]$, realizing K-theory in terms of D-branes $[9,10]$ and Myers effect [11-13] have been discovered.

In order to review string duality [14] is highly proposed. To observe information on the world volume of a $\mathrm{D}_{p}$-brane and in particular to deal with both Dirac-Born-Infeld and Chern-Simons effective actions we refer to [15-17] and all references therein. In order to encounter the effective action only for a bosonic $\mathrm{D}_{p}$-brane, [18] should be highlighted. It is widely understood that for multiple $\mathrm{D}_{p}$-branes, references $[11,13]$ are the main ones to look for bosonic action.

In order to see super symmetric action, one might search about some special references in [19] and [20-25].

Basically one has to emphasize the fact that the higher derivative corrections of stable and unstable branes are not involved in those effective actions, namely the only way for 
obtaining the closed form of all corrections is indeed scattering computations. To have all corrections, recent attempts in detail have been carried out. Morever, to discover the higher derivative corrections for stable branes, namely four field strengths' corrections to all orders in $\alpha^{\prime}$ [15] must be taken into account, also there we have shown that to $\alpha^{\prime 4}$ order computations are indeed consistent with literature [26-28]. To achieve two gauge field and two scalar fields ' corrections again to all orders of $\alpha^{\prime},[29]$ is suggested. Finally a pattern and a universal prescription for all BPS branes, including corrections to four covariant derivative of scalar fields has been found in [30].

Although in this paper we are interested in finding new couplings of gauge/scalars in the background of one closed string Ramond-Ramond field to all orders of $\alpha^{\prime}$, arguing effective actions for stable branes, addressing some recent works for unstable branes, such as [31] and [32] is highly recommended. In particular the effective action of brane-anti brane to all orders of $\alpha^{\prime}$ for two gauge and two tachyons have been derived in [33]. Recently remarks on the effective action of brane anti brane with all their $\alpha^{\prime}$ corrections in [34] have been made. Note that we do not review Wess-Zumino effective action here, but in order to follow all needed couplings, section 5 of [31] and references [5, 8] and [6] might be studied to pursue them for different values of $p$ and $n$, where $n$ is the rank of the field strength of the closed string Ramond-Ramond field.

Given several goals, involving dualities of Ads/CFT [35] and some exact relation between open and closed strings inside the Ads/CFT, examining new method for higher point string amplitudes is indeed necessary.

Two extremely important facts which must be really highlighted are:

1) In order to find all new couplings/contact interactions to all orders of $\alpha^{\prime}$ one has to have the complete form of the amplitudes. Note that the result of the amplitudes at leading orders is not very useful as we comment it in detail in this paper.

2) Once we are dealing with open-closed amplitudes T-duality transformation is not very effective and in fact direct computations of those amplitudes are inevitable. Even we want to work out tree level amplitude, the appearance of closed string RR makes the calculations so complicated. It is definitely realized that in loop computations applying T-duality is really subtle [36]. For example in [30] we have shown that it is not possible to derive $<V_{C} V_{\phi} V_{\phi} V_{\phi}>$ from $<V_{C} V_{A} V_{A} V_{\phi}>$. In particular we have seen that the terms including momentum of closed string $\mathrm{RR}$ in transverse directions $p^{i}, p^{j}$ are not appeared in $\left\langle V_{C} V_{A} V_{A} V_{\phi}>\right.$.

Now we address some of the motivations for the long computations of this paper. The first is to realize the closed form of new Wess-Zumino couplings to all orders in $\alpha^{\prime}$. It was argued in [31] that for the amplitudes including scalar fields and closed string Ramond-Ramond field, field theory vertices must be obtained just by three different methods, basically either through Myers' terms [11] or pull-back approach or Taylor expansion. However in this paper within detail we will show that there are some new couplings which do come from none of them. These new couplings for sure do not come from Myers' terms or Taylor expansion so this is a very strong evidence in favor of modifying pull-back (see also $[29,30])$. 
The other motivation is to get more data to see whether or not essentially we can write down the general form of DBI and Wess-Zumino effective actions. To check up to some orders [37-39] may be useful to look at.

The third reason for including $<V_{C} V_{A} V_{\phi} V_{\phi}>$ is indeed its direct relation to dielectric effect. As an example $N^{3}$ entropy of M5 branes was expressed in terms of dielectric effect in [40], for the other applications and for various configurations in M-theory see [41, 42]. Definitely the results of this paper on new couplings will provide fundamental steps for future outcomes such as all orders dielectric effect and various research topics on world volume dynamics of branes [35].

Two remarkable issues have been addressed. The first one is related to taking integrals for five point open-closed strings [43] in which for the first time has been applied in [33] and we were able to find several new couplings in the world volume of brane- anti brane systems. The second important fact is related to Wick-like formula [44]. Making use of this formula and by generalizing that in [15], we can now simply derive the correlation function between two spin operators in the presence of several currents and fermion fields. To deal with the integrations on some higher point functions, we suggest [45].

Dielectric effect does have various applications such as resolving some singularities in Ads/CFT by making use of closed string RR field to actually polarize $D_{3}$-brane (more details can be seen in [46-48]). The importance of this effect inside M-theory is argued in $[49,50]$, even it is more discussed in stabilizing the sources of $\mathrm{RR}$ in some particular backgrounds [51] relating to Wess-Zumino-Witten model, $A d s_{m} \times S^{n}$, fuzzy gravitons in some space-time $[52,53]$ or in terms of their gravity duals [54].

Given these applications, we are going to explore all new couplings between one closed string Ramond-Ramond field $(C)$ and some SYM vertex operators, namely three open strings (basically two scalars and one gauge field) in the IIB(A) super string theories. This $<V_{C} V_{A} V_{\phi} V_{\phi}>$ was introduced in [55] and in that paper making use of hyper geometric function the authors just were able to find the first simple pole of the amplitude, however, the complete form of the amplitude is unknown which we are going to find it out. Apart from that the authors in [55] have produced just the first scalar t-channel and the first gauge $(s+t+u)$-channel pole.

Using new techniques for five point amplitudes ( see appendix B of [31]) and making use of Wick-like formula we find the complete form of the amplitude of $\left\langle V_{C} V_{A} V_{\phi} V_{\phi}\right\rangle$. In addition to obtaining several new couplings to all orders of $\alpha^{\prime}$ which we will address in detail, we are going to show that the amplitude not only has gauge $\mathrm{u}$-channel pole but also it has infinite $\mathrm{u}$-channel poles which have been overlooked in [55] and could not be derived there because of not having the complete form of the amplitude.

Also, making use of the all order two gauge two scalar couplings that appeared in the very recent paper [29], we will find out the infinite gauge $(t+s+u)$-channel poles and infinite scalar $(t, s)$-channel poles as well.

Then we go on further and discover several new couplings for $p+2=n$ case. In particular by applying direct S-matrix computations and discovering the complete and closed form of the correlators of $<V_{C} V_{A} V_{\phi} V_{\phi}>$ we derive all new interactions to all orders in $\alpha^{\prime}$ for $p-2=n, p=n$ cases as well. 


\section{Complete form of the $C A \phi \phi$ amplitude to all orders of $\alpha^{\prime}$}

Here we are going to explore the S-matrix elements of another physically 4-point and technically 5-point function. Namely we do want to investigate in detail the amplitude of 3 BPS branes (2 scalar and one gauge fields) and one closed string RR field. Motivation for computing such a long computation is indeed checking all infinite couplings two gauge and two scalar fields which are recently discovered [29] and also trying to figure out how one can reproduce all infinite massless gauge and scalar fields for different values of $p$ and $n$.To compute a S-matrix element, one should clarify the picture of the vertex operators in an appropriate way. It would be nice to refer to some new works on BPS branes [29, $30]$ and [56].

Note that since we are looking for $<V_{C} V_{A} V_{\phi} V_{\phi}>$ some parts of the calculations are shared with $[15,29,55]$ but definitely all of the contact terms at all orders are new results and different from the previous results. It is important to highlight the following point. Due to $C$-field, it is not possible to obtain all contact terms of this paper from $<V_{C} V_{A} V_{A} V_{\phi}>$ by applying T-duality transformation, because we will observe here that all the terms involving $p^{i}, p^{j}$ are not present in $\left\langle V_{C} V_{A} V_{A} V_{\phi}>\right.$.

We must use the vertex operators according to the fact that for the disk level amplitudes (which we are dealing with) total charge has to be -2 .

Taking into account super ghost charges, we may write down the amplitude of one gauge, two open scalar fields and one closed string RR in terms of some kinds of special correlators

$$
\mathcal{A}^{C A \phi \phi} \sim \int d x_{1} d x_{2} d x_{3} d z d \bar{z}\left\langle V_{A}^{(0)}\left(x_{1}\right) V_{\phi}^{(0)}\left(x_{2}\right) V_{\phi}^{(0)}\left(x_{3}\right) V_{R R}^{\left(-\frac{3}{2},-\frac{1}{2}\right)}(z, \bar{z})\right\rangle,
$$

Since we are performing disk level amplitudes, all open strings must be put on the boundary of disk rather, RR has to be replaced in the middle of disk.

Depending on the various picture of the strings, One should pick up the following vertex operators. The vertex operators are known as ${ }^{1}$

$$
\begin{aligned}
V_{\phi}^{(0)}(x) & =\xi_{i}\left(\partial X^{i}(x)+\alpha^{\prime} i q \cdot \psi \psi^{i}(x)\right) e^{\alpha^{\prime} i q \cdot X(x)}, \\
V_{A}^{(0)}(x) & =\xi_{a}\left(\partial X^{a}(x)+\alpha^{\prime} i k \cdot \psi \psi^{a}(x)\right) e^{\alpha^{\prime} i k \cdot X(x)}, \\
V_{A}^{(-2)}(x) & =e^{-2 \phi(x)} V_{A}^{(0)}(x), \\
V_{A}^{(-1)}(y) & =\xi_{a} \psi^{a}(y) e^{-\phi(y)} e^{\alpha^{\prime} i k \cdot X(y)} \\
V_{R R}^{\left(-\frac{1}{2},-\frac{1}{2}\right)}(z, \bar{z}) & =\left(P_{-} H_{(n)} M_{p}\right)^{\alpha \beta} e^{-\phi(z) / 2} S_{\alpha}(z) e^{i \frac{\alpha^{\prime}}{2} p \cdot X(z)} e^{-\phi(\bar{z}) / 2} S_{\beta}(\bar{z}) e^{i \frac{\alpha^{\prime}}{2} p \cdot D \cdot X(\bar{z})}, \\
V_{R R}^{\left(-\frac{3}{2},-\frac{1}{2}\right)}(z, \bar{z}) & =\left(P_{-} \phi_{(n)} M_{p}\right)^{\alpha \beta} e^{-3 \phi(z) / 2} S_{\alpha}(z) e^{i \frac{\alpha^{\prime}}{2} p \cdot X(z)} e^{-\phi(\bar{z}) / 2} S_{\beta}(\bar{z}) e^{i \frac{\alpha^{\prime}}{2} p \cdot D \cdot X(\bar{z})},
\end{aligned}
$$

$q, k$ are scalar field and gauge field 's momenta which do satisfy the following conditions $k^{2}=q^{2}=0$ and $k_{i} \cdot \xi_{j}=0$. The definitions of projector and field strength of RR are

$$
H_{(n)}=\frac{a_{n}}{n !} H_{\mu_{1} \ldots \mu_{n}} \gamma^{\mu_{1}} \ldots \gamma^{\mu_{n}}, P_{-}=\frac{1}{2}\left(1-\gamma^{11}\right)
$$

\footnotetext{
${ }^{1}$ In string calculations, we used to set $\alpha^{\prime}=2$.
} 
$n$ is odd/even number for type IIB/IIA theory. To see more notation [15] is recommended. The simplest way to do this computation is as follows

$$
\mathcal{A}^{C A \phi \phi} \sim \int d x_{1} d x_{2} d x_{3} d z d \bar{z}\left\langle V_{A}^{(-1)}\left(x_{1}\right) V_{\phi}^{(0)}\left(x_{2}\right) V_{\phi}^{(0)}\left(x_{3}\right) V_{R R}^{\left(-\frac{1}{2},-\frac{1}{2}\right)}(z, \bar{z})\right\rangle
$$

Making use of the standard correlators for $X^{\mu}, \psi^{\mu}, \phi$ as follows

$$
\begin{aligned}
\left\langle X^{\mu}(z) X^{\nu}(w)\right\rangle & =-\frac{\alpha^{\prime}}{2} \eta^{\mu \nu} \log (z-w), \\
\left\langle\psi^{\mu}(z) \psi^{\nu}(w)\right\rangle & =-\frac{\alpha^{\prime}}{2} \eta^{\mu \nu}(z-w)^{-1}, \\
\langle\phi(z) \phi(w)\rangle & =-\log (z-w) .
\end{aligned}
$$

also introducing $x_{4} \equiv z=x+i y, x_{5} \equiv \bar{z}=x-i y$, the final form of the amplitude with just taking $\operatorname{Tr}\left(\lambda_{1} \lambda_{2} \lambda_{3}\right)$ ordering and with the closed form of the correlators reaches to

$$
\begin{array}{r}
\mathcal{A}^{C A \phi \phi} \sim \int d x_{1} d x_{2} d x_{3} d x_{4} d x_{5}\left(P_{-} H_{(n)} M_{p}\right)^{\alpha \beta} \xi_{1 a} \xi_{2 i} \xi_{3 j} x_{45}^{-1 / 4}\left(x_{14} x_{15}\right)^{-1 / 2} \\
\times\left(I_{1}+I_{2}+I_{3}+I_{4}\right) \operatorname{Tr}\left(\lambda_{1} \lambda_{2} \lambda_{3}\right),
\end{array}
$$

where $x_{i j}=x_{i}-x_{j}$. Having taken Wick theorem, one gets the correlators as

$$
\begin{gathered}
I_{1}=<: e^{\alpha^{\prime} i k_{1} \cdot X\left(x_{1}\right)}: \partial X^{i}\left(x_{2}\right) e^{\alpha^{\prime} i k_{2} \cdot X\left(x_{2}\right)}: \partial X^{j}\left(x_{3}\right) e^{\alpha^{\prime} i k_{3} \cdot X\left(x_{3}\right)}: e^{i \frac{\alpha^{\prime}}{2} p \cdot X\left(x_{4}\right)}: e^{i \frac{\alpha^{\prime}}{2} p \cdot D \cdot X\left(x_{5}\right)}:> \\
\times<: S_{\alpha}\left(x_{4}\right): S_{\beta}\left(x_{5}\right): \psi^{a}\left(x_{1}\right):> \\
I_{2}=<: e^{\alpha^{\prime} i k_{1} \cdot X\left(x_{1}\right)}: e^{\alpha^{\prime} i k_{2} \cdot X\left(x_{2}\right)}: \partial X^{j}\left(x_{3}\right) e^{\alpha^{\prime} i k_{3} \cdot X\left(x_{3}\right)}: e^{i \frac{\alpha^{\prime}}{2} p \cdot X\left(x_{4}\right)}: e^{i \frac{\alpha^{\prime}}{2} p \cdot D \cdot X\left(x_{5}\right)}:> \\
\times<: S_{\alpha}\left(x_{4}\right): S_{\beta}\left(x_{5}\right):: \psi^{a}\left(x_{1}\right): \alpha^{\prime} i k_{2} \cdot \psi \psi^{i}\left(x_{2}\right)> \\
I_{3}=<: e^{\alpha^{\prime} i k_{1} \cdot X\left(x_{1}\right)}: \partial X^{i}\left(x_{2}\right) e^{\alpha^{\prime} i k_{2} \cdot X\left(x_{2}\right)}: e^{\alpha^{\prime} i k_{3} \cdot X\left(x_{3}\right)}: e^{i \frac{\alpha^{\prime}}{2} p \cdot X\left(x_{4}\right)}: e^{i \frac{\alpha^{\prime}}{2} p \cdot D \cdot X\left(x_{5}\right)}:> \\
\times<: S_{\alpha}\left(x_{4}\right): S_{\beta}\left(x_{5}\right):: \psi^{a}\left(x_{1}\right): \alpha^{\prime} i k_{3} \cdot \psi \psi^{j}\left(x_{3}\right)>, \\
I_{4}=<: e^{\alpha^{\prime} i k_{1} \cdot X\left(x_{1}\right)}: e^{\alpha^{\prime} i k_{2} \cdot X\left(x_{2}\right)}: e^{\alpha^{\prime} i k_{3} \cdot X\left(x_{3}\right)}: e^{i \frac{\alpha^{\prime}}{2} p \cdot X\left(x_{4}\right)}: e^{i \frac{\alpha^{\prime}}{2} p \cdot D \cdot X\left(x_{5}\right)}:> \\
\times \\
\times
\end{gathered}
$$

Needless to remind that the following correlation has been achieved by working out the generalized form of Wick-like [15]

$$
\begin{aligned}
I_{5}^{i b a}= & <: S_{\alpha}\left(x_{4}\right): S_{\beta}\left(x_{5}\right): \psi^{a}\left(x_{1}\right): \psi^{b} \psi^{i}\left(x_{2}\right):> \\
= & \left\{\left(\Gamma^{i b a} C^{-1}\right)_{\alpha \beta}+\frac{\alpha^{\prime} \operatorname{Re}\left[x_{14} x_{25}\right]}{x_{12} x_{45}}\left(-\eta^{a b}\left(\gamma^{i} C^{-1}\right)_{\alpha \beta}\right)\right\} \\
& \times 2^{-3 / 2} x_{45}^{1 / 4}\left(x_{24} x_{25}\right)^{-1}\left(x_{14} x_{15}\right)^{-1 / 2} .
\end{aligned}
$$


Having used the arguments mentioned in [15], the following correlator can be easily gained:

$$
\begin{aligned}
I_{6}^{j c i b a}=<: & S_{\alpha}\left(x_{4}\right): S_{\beta}\left(x_{5}\right):: \psi^{a}\left(x_{1}\right): \psi^{b} \psi^{i}\left(x_{2}\right): \psi^{c} \psi^{j}\left(x_{3}\right)> \\
= & \left\{\left(\Gamma^{j c i b a} C^{-1}\right)_{\alpha \beta}+\alpha^{\prime} r_{1} \frac{\operatorname{Re}\left[x_{14} x_{25}\right]}{x_{12} x_{45}}+\alpha^{\prime} r_{2} \frac{\operatorname{Re}\left[x_{14} x_{35}\right]}{x_{13} x_{45}}+\alpha^{\prime} r_{3} \frac{\operatorname{Re}\left[x_{24} x_{35}\right]}{x_{23} x_{45}}\right. \\
& +\left(\alpha^{\prime 2}\right) r_{4}\left(\frac{\operatorname{Re}\left[x_{24} x_{35}\right]}{x_{23} x_{45}}\right)^{2}+\left(\alpha^{\prime 2}\right) r_{5}\left(\frac{\operatorname{Re}\left[x_{14} x_{25}\right]}{x_{12} x_{45}} \times \frac{\operatorname{Re}\left[x_{24} x_{35}\right]}{x_{23} x_{45}}\right) \\
& \left.\quad+\left(\alpha^{\prime 2}\right) r_{6}\left(\frac{\operatorname{Re}\left[x_{14} x_{35}\right]}{x_{13} x_{45}} \frac{\operatorname{Re}\left[x_{24} x_{35}\right]}{x_{23} x_{45}}\right)\right\} 2^{-5 / 2} x_{45}^{5 / 4}\left(x_{24} x_{25} x_{34} x_{35}\right)^{-1}\left(x_{14} x_{15}\right)^{-1 / 2},
\end{aligned}
$$

where

$$
\begin{aligned}
& r_{1}=\left(-\eta^{a b}\left(\Gamma^{j c i} C^{-1}\right)_{\alpha \beta}\right), \\
& r_{2}=\left(-\eta^{a c}\left(\Gamma^{j i b} C^{-1}\right)_{\alpha \beta}\right), \\
& r_{3}=\left(\eta^{b c}\left(\Gamma^{j i a} C^{-1}\right)_{\alpha \beta}+\eta^{i j}\left(\Gamma^{c b a} C^{-1}\right)_{\alpha \beta}\right), \\
& r_{4}=\left(\left(-\eta^{b c} \eta^{i j}\right)\left(\gamma^{a} C^{-1}\right)_{\alpha \beta}\right), \\
& r_{5}=\left(\left(-\eta^{a b} \eta^{i j}\right)\left(\gamma^{c} C^{-1}\right)_{\alpha \beta}\right), \\
& r_{6}=\left(\left(\eta^{a c} \eta^{i j}\right)\left(\gamma^{b} C^{-1}\right)_{\alpha \beta}\right) .
\end{aligned}
$$

Having regarded all those correlators in our amplitude, we find the closed form of these S-matrix elements as:

$$
\begin{aligned}
\mathcal{A}^{C A \phi \phi} \sim \int d x_{1} d x_{2} d x_{3} d x_{4} d x_{5}\left(P_{-} H_{(n)} M_{p}\right)^{\alpha \beta} I \xi_{1 a} \xi_{2 i} \xi_{3 j} x_{45}^{-1 / 4}\left(x_{14} x_{15}\right)^{-1 / 2} \\
\times\left(I_{7}^{a}\left(-\eta^{i j} x_{23}^{-2}+a_{1}^{j} a_{2}^{i}\right)+a_{1}^{j} a_{3}^{i a}+a_{2}^{i} a_{4}^{j a}-\alpha^{\prime 2} k_{2 b} k_{3 c} I_{6}^{j c i b a}\right) \operatorname{Tr}\left(\lambda_{1} \lambda_{2} \lambda_{3}\right),
\end{aligned}
$$

where $I_{6}^{j c i b a}$ is given in $(2.8)$ and

$$
\begin{aligned}
& I=\left|x_{12}\right|^{\alpha^{\prime 2} k_{1} \cdot k_{2}}\left|x_{13}\right|^{\alpha^{\prime 2} k_{1} \cdot k_{3}}\left|x_{14} x_{15}\right|^{\frac{\alpha^{\prime 2}}{2} k_{1} \cdot p}\left|x_{23}\right|^{\alpha^{\prime 2} k_{2} \cdot k_{3}} \times \\
& \times\left|x_{24} x_{25}\right|^{\frac{\alpha^{\prime 2}}{2} k_{2} \cdot p}\left|x_{34} x_{35}\right|^{\frac{\alpha^{\prime 2}}{2} k_{3} \cdot p}\left|x_{45}\right|^{\frac{\alpha^{\prime 2}}{4} p . D \cdot p}, \\
& a_{1}^{j}=i p^{j} \frac{x_{54}}{x_{34} x_{35}} \\
& a_{2}^{i}=i p^{i} \frac{x_{54}}{x_{24} x_{25}}, \\
& a_{3}^{i a}=\alpha^{\prime} i k_{2 b} I_{5}^{i b a}, \\
& a_{4}^{j a}=\alpha^{\prime} i k_{3 c} 2^{-3 / 2} x_{45}^{1 / 4}\left(x_{34} x_{35}\right)^{-1}\left(x_{14} x_{15}\right)^{-1 / 2} \\
& \times\left\{\left(\Gamma^{j c a} C^{-1}\right)_{\alpha \beta}+\frac{\alpha^{\prime} R e\left[x_{14} x_{35}\right]}{x_{13} x_{45}}\left(-\eta^{a c}\left(\gamma^{j} C^{-1}\right)_{\alpha \beta}\right)\right\}, \\
& I_{7}^{a}=<: S_{\alpha}\left(x_{4}\right): S_{\beta}\left(x_{5}\right): \psi^{a}\left(x_{1}\right):>=2^{-1 / 2} x_{45}^{-3 / 4}\left(x_{14} x_{15}\right)^{-1 / 2}\left(\gamma^{a} C^{-1}\right)_{\alpha \beta} .
\end{aligned}
$$


Now we are ready to show that the amplitude is written such that $\mathrm{SL}(2, R)$ transformation holds.

We apply a special gauge fixing which is different from the ones that appeared in [55], that is why we can find out the general form of the amplitude. Basically we just fixed the positions of all three massless open strings in $(0,1, \infty)$ and carry out all integrations by making use of the integrals obtained in [31]. The other fact which has been widely used is indeed introducing the following Mandelstam variables

$$
s=-\frac{\alpha^{\prime}}{2}\left(k_{1}+k_{3}\right)^{2}, \quad t=-\frac{\alpha^{\prime}}{2}\left(k_{1}+k_{2}\right)^{2}, \quad u=-\frac{\alpha^{\prime}}{2}\left(k_{2}+k_{3}\right)^{2} .
$$

Eventually we try to find out the final form of the amplitude (2.10) as

$$
\mathcal{A}^{C A \phi \phi}=\mathcal{A}_{1}+\mathcal{A}_{2}+\mathcal{A}_{3}+\mathcal{A}_{4}+\mathcal{A}_{5}+\mathcal{A}_{6}+\mathcal{A}_{7}+\mathcal{A}_{8}+\mathcal{A}_{9}+\mathcal{A}_{10}
$$

where

$$
\begin{aligned}
& \mathcal{A}_{1} \sim-2^{-1 / 2} \xi_{1 a} \xi_{2 i} \xi_{3 j}\left[k_{3 c} k_{2 b} \operatorname{Tr}\left(P_{-} H_{(n)} M_{p} \Gamma^{j c i b a}\right)-k_{2 b} p^{j} \operatorname{Tr}\left(P_{-} H_{(n)} M_{p} \Gamma^{i b a}\right)\right.\left.-k_{3 c} p^{i} \operatorname{Tr}\left(P_{-} H_{(n)} M_{p} \Gamma^{j c a}\right)+p^{i} p^{j} \operatorname{Tr}\left(P_{-} H_{(n)} M_{p} \gamma^{a}\right)\right] L_{1}, \\
& \mathcal{A}_{2} \sim 2^{-1 / 2}\left\{-2 \xi_{1} \cdot k_{2} k_{3 c} \xi_{3 j} \xi_{2 i} \operatorname{Tr}\left(P_{-} H_{(n)} M_{p} \Gamma^{j c i}\right)\right\} L_{2} \\
& \mathcal{A}_{3} \sim 2^{-1 / 2}\left\{\xi_{1 a} \xi_{2 i} \xi_{3 j} \operatorname{Tr}\left(P_{-} H_{(n)} M_{p} \Gamma^{j i a}\right)\right\} L_{22} \\
& \mathcal{A}_{4} \sim 2^{-1 / 2}\left\{2 k_{3} \cdot \xi_{1} k_{2 b} \xi_{3 j} \xi_{2 i} \operatorname{Tr}\left(P_{-} H_{(n)} M_{p} \Gamma^{j i b}\right)\right\} L_{3} \\
& \mathcal{A}_{5} \sim 2^{-1 / 2}\left\{2 \xi_{3} \cdot \xi_{2} k_{2 b} k_{3 c} \xi_{1 a} \operatorname{Tr}\left(P_{-} H_{(n)} M_{p} \Gamma^{c b a}\right)\right\} L_{5} \\
& \mathcal{A}_{6} \sim 2^{1 / 2} L_{2}\left\{p^{j} \xi_{1} \cdot k_{2} \xi_{2 i} \xi_{3 j} \operatorname{Tr}\left(P_{-} H_{(n)} M_{p} \gamma^{i}\right)\right\} \\
& \mathcal{A}_{7} \sim-2^{-1 / 2} L_{3}\left\{2 k_{3} \cdot \xi_{1} p^{i} \xi_{3 j} \xi_{2 i} \operatorname{Tr}\left(P_{-} H_{(n)} M_{p} \gamma^{j}\right)\right\} \\
& \mathcal{A}_{8} \sim 2^{1 / 2} L_{6}\left\{2 k_{2} \cdot \xi_{1} k_{3 c} \operatorname{Tr}\left(P_{-} H_{(n)} M_{p} \gamma^{c}\right)\left(-s \xi_{2} \cdot \xi_{3}\right)\right\} . \\
& \mathcal{A}_{9} \sim 2^{1 / 2} L_{6}\left\{2 k_{3} \cdot \xi_{1} k_{2 b} \operatorname{Tr}\left(P_{-} H_{(n)} M_{p} \gamma^{b}\right)\left(-t \xi_{2} \cdot \xi_{3}\right)\right\} \\
& \mathcal{A}_{10} \sim 2^{1 / 2} L_{6}\left\{\xi_{1 a} \operatorname{Tr}\left(P_{-} H_{(n)} M_{p} \gamma^{a}\right)\left(t s \xi_{3} \cdot \xi_{2}\right)\right\}
\end{aligned}
$$

where the functions $L_{1}, L_{2}, L_{22}, L_{3}, L_{5}, L_{6}$ are appeared in the following

$$
\begin{aligned}
L_{1} & =(2)^{-2(t+s+u)+1} \pi \frac{\Gamma\left(-u+\frac{1}{2}\right) \Gamma\left(-s+\frac{1}{2}\right) \Gamma\left(-t+\frac{1}{2}\right) \Gamma(-t-s-u+1)}{\Gamma(-u-t+1) \Gamma(-t-s+1) \Gamma(-s-u+1)}, \\
L_{2} & =(2)^{-2(t+s+u)} \pi \frac{\Gamma(-u+1) \Gamma(-s+1) \Gamma(-t) \Gamma\left(-t-s-u+\frac{1}{2}\right)}{\Gamma(-u-t+1) \Gamma(-t-s+1) \Gamma(-s-u+1)} \\
L_{22} & =(2)^{-2(t+s+u)} \pi \frac{\Gamma(-u+1) \Gamma(-s+1) \Gamma(-t+1) \Gamma\left(-t-s-u+\frac{1}{2}\right)}{\Gamma(-u-t+1) \Gamma(-t-s+1) \Gamma(-s-u+1)} \\
L_{3} & =(2)^{-2(t+s+u)} \pi \frac{\Gamma(-u+1) \Gamma(-s) \Gamma(-t+1) \Gamma\left(-t-s-u+\frac{1}{2}\right)}{\Gamma(-u-t+1) \Gamma(-t-s+1) \Gamma(-s-u+1)},
\end{aligned}
$$




$$
\begin{aligned}
& L_{5}=(2)^{-2(t+s+u)} \pi \frac{\Gamma(-u) \Gamma(-s+1) \Gamma(-t+1) \Gamma\left(-t-s-u+\frac{1}{2}\right)}{\Gamma(-u-t+1) \Gamma(-t-s+1) \Gamma(-s-u+1)}, \\
& L_{6}=(2)^{-2(t+s+u)-1} \pi \frac{\Gamma\left(-u+\frac{1}{2}\right) \Gamma\left(-s+\frac{1}{2}\right) \Gamma\left(-t+\frac{1}{2}\right) \Gamma(-t-s-u)}{\Gamma(-u-t+1) \Gamma(-t-s+1) \Gamma(-s-u+1)},
\end{aligned}
$$

We could actually simplify the final result more as follows:

$$
\mathcal{A}^{C A \phi \phi}=\mathcal{A}_{1}+\mathcal{A}_{2}+\mathcal{A}_{3}+\mathcal{A}_{4}
$$

where

$$
\begin{aligned}
& \mathcal{A}_{1} \sim 2^{-1 / 2} 4 \xi_{1 a} \xi_{2 i} \xi_{3 j}(t+s+u) L_{1}^{\prime}\left[k_{3 c} k_{2 b} \operatorname{Tr}\left(P_{-} H_{(n)} M_{p} \Gamma^{j c i b a}\right)-k_{2 b} p^{j} \operatorname{Tr}\left(P_{-} H_{(n)} M_{p} \Gamma^{i b a}\right)\right. \\
& \left.-k_{3 c} p^{i} \operatorname{Tr}\left(P_{-} H_{(n)} M_{p} \Gamma^{j c a}\right)+p^{i} p^{j} \operatorname{Tr}\left(P_{-} H_{(n)} M_{p} \gamma^{a}\right)\right] \\
& \mathcal{A}_{2} \sim 2^{-1 / 2} L_{2}^{\prime}\left\{-2 u s \xi_{1} . k_{2} \xi_{2 i} k_{3 c} \xi_{3 j} \operatorname{Tr}\left(P_{-} H_{(n)} M_{p} \Gamma^{j c i}\right)-u s t \xi_{1 a} \xi_{2 i} \xi_{3 j} \operatorname{Tr}\left(P_{-} H_{(n)} M_{p} \Gamma^{j i a}\right)\right. \\
& +2 u t k_{3} \cdot \xi_{1} k_{2 b} \xi_{3 j} \xi_{2 i} \operatorname{Tr}\left(P_{-} H_{(n)} M_{p} \Gamma^{j i b}\right)+2 u s \xi_{2 i} p^{j} \xi_{1} \cdot k_{2} \xi_{3 j} \operatorname{Tr}\left(P_{-} H_{(n)} M_{p} \gamma^{i}\right) \\
& \left.-2 u t \xi_{3 j} p^{i} \xi_{1} \cdot k_{3} \xi_{2 i} \operatorname{Tr}\left(P_{-} H_{(n)} M_{p} \gamma^{j}\right)\right\} \\
& \mathcal{A}_{3} \sim 2^{1 / 2} \operatorname{Tr}\left(P_{-} H_{(n)} M_{p} \gamma^{a}\right) \xi_{3} \cdot \xi_{2} L_{1}^{\prime}\left[t s \xi_{1 a}-2 t k_{3} \cdot \xi_{1} k_{2 a}-2 s k_{2} \cdot \xi_{1} k_{3 a}\right] \\
& \mathcal{A}_{4} \sim 2^{-1 / 2} L_{2}^{\prime}\left\{2 s t \xi_{3} \cdot \xi_{2} k_{2 b} k_{3 c} \xi_{1 a} \operatorname{Tr}\left(P_{-} H_{(n)} M_{p} \Gamma^{c b a}\right)\right\} \text {. }
\end{aligned}
$$

where the functions $L_{1}^{\prime}, L_{2}^{\prime}$ now defined as

$$
\begin{aligned}
& L_{1}^{\prime}=(2)^{-2(t+s+u)-1} \pi \frac{\Gamma\left(-u+\frac{1}{2}\right) \Gamma\left(-s+\frac{1}{2}\right) \Gamma\left(-t+\frac{1}{2}\right) \Gamma(-t-s-u)}{\Gamma(-u-t+1) \Gamma(-t-s+1) \Gamma(-s-u+1)}, \\
& L_{2}^{\prime}=(2)^{-2(t+s+u)} \pi \frac{\Gamma(-u) \Gamma(-s) \Gamma(-t) \Gamma\left(-t-s-u+\frac{1}{2}\right)}{\Gamma(-u-t+1) \Gamma(-t-s+1) \Gamma(-s-u+1)}
\end{aligned}
$$

One important test of our amplitude is indeed applying Ward identity. Replacing $\xi_{1 a} \rightarrow k_{1 a}$, one believes that all parts of amplitude become zero. Notice that our amplitude makes sense for $n=p-2, n=p+2$ and $p=n$ cases.

Given the facts that we are dealing with 5-point super string computations for all massless strings and we are applying momentum conservation just for longitudinal direction, it is expected to get the same relation as appeared in [15, 29]. Thus the following relation holds

$$
s+t+u=-p_{a} p^{a} .
$$

As argued in [30], the expansion must be done by sending all three Mandelstam variables to zero.

It is worth taking the fact that both $L_{1}^{\prime}, L_{2}^{\prime}$ are symmetrized in terms of $(u, t, s)$ and this provides some confusions to indeed derive the general form of our expansions, however the field theory is a very useful guide in order for obtaining desired expansions. Since we are carrying out technically 5-point function for all massless particles, the expansions and the coefficients are the same as appeared in the amplitude of one Ramond-Ramond and 
three massless scalar fields [30], however, the terms that appeared in those amplitudes are really different from the terms of $<V_{C} V_{A} V_{\phi} V_{\phi}>$ and this is one the reasons for performing explicit computations. The corrected expansions are

$$
\begin{gathered}
L_{1}^{\prime}=-\frac{\pi^{5 / 2}}{2}\left(\sum_{n=0}^{\infty} c_{n}(s+t+u)^{n}+\frac{\sum_{n, m=0}^{\infty} c_{n, m}\left[s^{n} t^{m}+s^{m} t^{n}\right]}{(t+s+u)}\right. \\
\left.+\sum_{p, n, m=0}^{\infty} f_{p, n, m}(s+t+u)^{p}\left[(s+t)^{n}(s t)^{m}\right]\right), \\
s u L_{2}^{\prime}=-\pi^{3 / 2}\left(\sum_{n=-1}^{\infty} b_{n}\left(\frac{1}{t}(u+s)^{n+1}\right)+\sum_{p, n, m=0}^{\infty} e_{p, n, m} t^{p}(s u)^{n}(s+u)^{m}\right)
\end{gathered}
$$

Finally in order to obtain the suitable expansions for $s t L_{2}^{\prime}$ and $t u L_{2}^{\prime}$, one must replace $t \leftrightarrow u$ in (2.20) and $t \leftrightarrow s$ accordingly such that

$$
\begin{aligned}
& t u L_{2}^{\prime}=-\pi^{3 / 2}\left(\sum_{n=-1}^{\infty} b_{n}\left(\frac{1}{s}(u+t)^{n+1}\right)+\sum_{p, n, m=0}^{\infty} e_{p, n, m} s^{p}(t u)^{n}(t+u)^{m}\right) \\
& t s L_{2}^{\prime}=-\pi^{3 / 2}\left(\sum_{n=-1}^{\infty} b_{n}\left(\frac{1}{u}(s+t)^{n+1}\right)+\sum_{p, n, m=0}^{\infty} e_{p, n, m} u^{p}(t s)^{n}(t+s)^{m}\right)
\end{aligned}
$$

In order to produce all massless poles for different values of $p$ and $n$ one has to know some of the coefficients in those expansions:

$$
\begin{aligned}
& b_{-1}=1, \quad b_{0}=0, \quad b_{1}=\frac{1}{6} \pi^{2}, \quad b_{2}=2 \zeta(3), \\
& c_{0}=0, \quad c_{1}=\frac{\pi^{2}}{6}, \quad e_{0,0,1}=\frac{1}{3} \pi^{2}, \\
& e_{2,0,0}=e_{0,1,0}=2 \zeta(3), \quad e_{1,0,0}=\frac{1}{6} \pi^{2}, \quad e_{1,0,2}=\frac{19}{60} \pi^{4}, \quad e_{1,0,1}=e_{0,0,2}=6 \zeta(3), \\
& c_{2}=-2 \zeta(3), \quad c_{1,1}=\frac{\pi^{2}}{6}, \quad c_{0,0}=\frac{1}{2}, \\
& c_{3,1}=c_{1,3}=\frac{2}{15} \pi^{4}, \quad c_{2,2}=\frac{1}{5} \pi^{4}, \quad f_{0,1,0}=-\frac{1}{3} \pi^{2} \\
& c_{1,0}=c_{0,1}=0, \quad c_{3,0}=c_{0,3}=0, \quad c_{2,0}=c_{0,2}=\frac{\pi^{2}}{6}, \\
& c_{1,2}=c_{2,1}=-4 \zeta(3), \quad c_{4,0}=c_{0,4}=\frac{1}{15} \pi^{4}
\end{aligned}
$$

The important point here is that, $L_{1}^{\prime}$ for our amplitude $<V_{C} V_{A} V_{\phi} V_{\phi}>$ must have infinite massless gauge but not scalar poles in the $(t+s+u)$-channel and this is unlike $<V_{C} V_{A} V_{A} V_{\phi}>$, which had infinite massless scalar poles. The other point which must be mentioned before carrying out field theory computations is that, $L_{2}^{\prime}$ must have either infinite massless scalar poles in $t, s$ channels or infinite massless gauge poles in $u$-channels which we take care of them in a closed form in the next sections. 


\subsection{Infinite massless gauge poles for $p-2=n$ case}

In this section, we are going to explore all infinite $\mathrm{u}$-channel gauge poles with new WessZumino couplings. These poles have been overlooked in [55]. By applying $s t L_{2}^{\prime}$ expansion into $\mathcal{A}_{4}$ amplitude in (2.15) one should have got all massless gauge poles in string amplitude as

$$
-\pi^{2} \mu_{p}\left(2 \xi_{3} \cdot \xi_{2} k_{2 b} k_{3 c} \xi_{1 a}\right) \frac{16}{(p-2) !} \epsilon^{a_{0} \cdots a_{p-3} c b a} H_{a_{0} \cdots a_{p-3}} \sum_{n=-1}^{\infty} b_{n}\left(\frac{1}{u}(t+s)^{n+1}\right) \operatorname{Tr}\left(\lambda_{1} \lambda_{2} \lambda_{3}\right)
$$

where trace has been taken and we just kept all poles in the expansion of $s t L_{2}^{\prime}$. Note that we normalized the amplitude by multiplying a coefficient of $2^{1 / 2} \pi^{1 / 2} \mu_{p}$.

The important point should be highlighted is that, (2.24) has been anti symmetrized in terms of both scalars and this leads to the conclusion that amplitude must be non-vanished just for non Abelian gauge group.

One test of this part of amplitude is, taking into account Ward identity for the gauge field. Thus by replacing $\xi_{1 a} \rightarrow k_{1 a}$, making use of momentum conservation and applying physical state condition for $\mathrm{RR}\left(p^{a} \epsilon^{a_{0} \cdots a_{p-3} c b a}=0\right)$, we observe that $(2.24)$ does vanish.

The related Feynman rule in field theory side for $p-2=n$ case is

$$
\mathcal{A}=V_{\alpha}^{a}\left(C_{p-3}, A_{1}, A\right) G_{\alpha \beta}^{a b}(A) V_{\beta}^{b}\left(A, \phi_{2}, \phi_{3}\right),
$$

The needed vertex $V_{\alpha}^{a}\left(C_{p-3}, A_{1}, A\right)$ in field theory must be obtained by taking this Chern-Simons coupling

$$
S_{1}=i \lambda^{2} \mu_{p} \int d^{p+1} \sigma \quad \operatorname{Tr}\left(C_{(p-3)} \wedge F \wedge F\right)
$$

Note that in the above action $F_{a b}=\partial^{a} A^{b}-\partial^{b} A^{a}-i\left[A^{a}, A^{b}\right]$ and $\lambda=2 \pi \alpha^{\prime}$, however all commutators must be neglected as we are looking for the coupling between one RR- $(p-3)$ form and two gaue fields. Integration by parts are also taken such that

$$
V_{\alpha}^{a}\left(C_{p-3}, A_{1}, A\right)=\lambda^{2} \mu_{p} \frac{1}{(p-2) !} \epsilon^{a_{0} \cdots a_{p-1} a} H_{a_{0} \cdots a_{p-3}} \xi_{1 a_{p-2}} k_{a_{p-1}}
$$

As it becomes clear from (2.24) the amplitude has infinite poles. The vertex of $V_{\beta}^{b}\left(A, \phi_{2}, \phi_{3}\right)$ should be derived from the kinetic term of scalar fields in DBI action $\left[\frac{\lambda^{2}}{2} \operatorname{Tr}\left(D^{a} \phi^{i} D_{a} \phi_{i}\right)\right]$ as follows

$$
\begin{aligned}
V_{\beta}^{b}\left(A, \phi_{2}, \phi_{3}\right) & =i \lambda^{2} T_{p} \xi_{2} \cdot \xi_{3}\left(k_{2}-k_{3}\right)^{b} \operatorname{Tr}\left(\lambda_{2} \lambda_{3} \lambda_{\beta}\right) \\
G_{\alpha \beta}^{a b}(\phi) & =\frac{-i}{\lambda^{2} T_{p}} \frac{\delta^{a b} \delta_{\alpha \beta}}{k^{2}}
\end{aligned}
$$

Consider $k^{2}=-\left(k_{2}+k_{3}\right)^{2}=u$ in the above propagator.

Kinetic term of scalar field indeed has been fixed so definitely there is no correction to all kinetic terms such as kinetic term of scalars. Also notice that massless poles here are simple massless poles thus neither do they get corrected. In addition to that, by considering (2.26) we could produce just the first simple gauge pole out of infinite poles. 
Having got this remarkable fact, we come to the point that, in order to produce all infinite gauge poles one has to find out all related corrections to $i \lambda^{2} \mu_{p} \int d^{p+1} \sigma C_{(p-3)} \wedge F \wedge F$ as

$$
S_{2}=i \lambda^{2} \mu_{p} \int d^{p+1} \sigma \quad \sum_{n=-1}^{\infty} b_{n}\left(\alpha^{\prime}\right)^{n+1} \quad C_{(p-3)} \wedge D_{a_{0} \cdots a_{n}} F \wedge D^{a_{0} \cdots a_{n}} F
$$

for more explanations see [15]. By setting these corrections, we are indeed able to produce all massless gauge poles to all orders of $\alpha^{\prime}$. Let us write down the corrected form of the needed vertex to all orders as

$$
\begin{aligned}
V_{\alpha}^{a}\left(C_{p-3}, A_{1}, A\right)=\frac{\lambda^{2} \mu_{p}}{(p-2) !}(\epsilon)^{a_{0} \cdots a_{p-1} a}\left(H^{(p-2)}\right)_{a_{0} \cdots a_{p-3}} \xi_{1 a_{p-2}} k_{a_{p-1}} \\
\times \operatorname{Tr}\left(\lambda_{1} \lambda_{\alpha}\right) \sum_{n=-1}^{\infty} b_{n}\left(\alpha^{\prime} k_{1} \cdot k\right)^{n+1}
\end{aligned}
$$

Having replaced $(2.29),(2.27)$ into (2.25) we may write down the result as

$$
\begin{aligned}
& \mathcal{A}=\mu_{p}\left(2 \pi \alpha^{\prime}\right)^{2} \frac{1}{(p-2) ! u} \operatorname{Tr}\left(\lambda_{1} \lambda_{2} \lambda_{3}\right) \epsilon^{a_{0} \cdots a_{p-1} a} H_{a_{0} \cdots a_{p-3}} \xi_{1 a_{p-2}} \times \\
& \times \sum_{n=-1}^{\infty} b_{n}\left(\frac{\alpha^{\prime}}{2}\right)^{n+1}(s+t)^{n+1}\left[2 k_{2 a} k_{3 a_{p-1}} \xi_{2} \cdot \xi_{3}\right]
\end{aligned}
$$

Thus (2.30) can exactly produce all ifinite massless gauge poles which we were looking for in (2.24). Indeed we have precisely produced all $\mathrm{u}$-channel poles in this section. This is the other new result of this paper.

\section{New couplings for BPS-branes for $n=p-2$ case}

In this section, by comparing direct result of string amplitude, we are going to discover new Wess-Zumino couplings for $n=p-2$ case at leading order and generalize them to actually construct all their higher order corrections as well.

To start, we rewrite the explicit form of string amplitude for this case as

$$
A_{4}=2 \pi^{1 / 2} \mu_{p} s t \xi_{3} \cdot \xi_{2} k_{2 b} k_{3 c} \xi_{1 a} \operatorname{Tr}\left(P_{-} H_{(n)} M_{p} \Gamma^{c b a}\right) L_{2}^{\prime}
$$

Extracting the trace and applying $s t L_{2}^{\prime}$ expansion we get

$$
\begin{aligned}
A_{4}=-2 \xi_{3} \cdot \xi_{2} k_{2 b} k_{3 c} \xi_{1 a} \pi^{2} \mu_{p} \frac{16}{(p-2) !} \epsilon^{a_{0} \cdots a_{p-3} c b a} H_{a_{0} \cdots a_{p-3}} \\
\times\left(\sum_{n=-1}^{\infty} b_{n}\left(\frac{1}{u}(t+s)^{n+1}\right)+\sum_{p, n, m=0}^{\infty} e_{p, n, m} u^{p}(s t)^{n}(s+t)^{m}\right)
\end{aligned}
$$

In the last section, and in particular in (2.30), comparing with string theory amplitude, we have produced all infinite $\mathrm{u}$-channel poles in field theory. Now in order for gaining new 
couplings with exact coefficients, what we have to take into account is indeed the second term in (3.1), that is,

$$
\begin{aligned}
\left.A_{4}^{C A \phi \phi}=-2 \xi_{3} \cdot \xi_{2} k_{2 b} k_{3 c} \xi_{1 a} \pi^{2} \mu_{p} \frac{16}{(p}-2\right) ! & \epsilon^{a_{0} \cdots a_{p-3} c b a} H_{a_{0} \cdots a_{p-3}} \\
& \times\left(\sum_{p, n, m=0}^{\infty} e_{p, n, m} u^{p}(s t)^{n}(s+t)^{m}\right)
\end{aligned}
$$

Note that (3.2) by itself does satisfy Ward identity, namely if we replace $\xi_{1 a}$ to $k_{1 a}$ and apply the momentum conservation along the world volume and in particular consider the physical state condition for the $\mathrm{RR} p^{a} \epsilon^{a_{0} \cdots a_{p-1} a}=0$, we come to the fact that this part of the amplitude should be written just in terms of a new Wess-Zumino coupling which must have the following structure

$$
\int_{\sum_{p+1}} d^{p+1} \sigma \quad \operatorname{Tr}\left(C_{p-3} \wedge F \wedge D \phi^{i} \wedge D \phi_{i}\right)
$$

It is written such that, it covers the world volume space and also satisfies antisymmetrization with respect to the interchange of scalar field's momenta. Let us apply $e_{1,0,0}=\frac{\pi^{2}}{6}$ and $e_{0,0,1}=\frac{\pi^{2}}{3}$ to (3.3) and produce the first non-zero couplings as $S_{3}$ and $S_{4}$ then generalize all orders in $\alpha^{\prime}$ higher derivative corrections:

$$
\begin{aligned}
S_{3}=\frac{\lambda^{3} \mu_{p} \pi}{12} \int d^{p+1} & \sigma \frac{1}{(p-3) !}\left(\varepsilon^{v}\right)^{a_{0} \cdots a_{p}}\left(\frac{\alpha^{\prime}}{2}\right) \\
& \times C_{a_{0} \cdots a_{p-4}}^{(p-3)} \operatorname{Tr}\left(F_{a_{p-3} a_{p-2}}\left(D^{a} D_{a}\right)\left[D_{a_{p-1}} \phi^{i} D_{a_{p}} \phi_{i}\right]\right)
\end{aligned}
$$

and

$$
S_{4}=\frac{\lambda^{3} \mu_{p} \pi}{6} \int d^{p+1} \sigma\left(\alpha^{\prime}\right) \operatorname{Tr}\left(C_{p-3} \wedge D^{b_{1}} F \wedge D_{b_{1}}\left[D \phi^{i} \wedge D \phi_{i}\right]\right)
$$

It is not difficult to investigate that, in order to produce (3.2), the closed form of higher derivative corrections to all orders of $\alpha^{\prime}$ must be taken as follows

$$
\begin{aligned}
S_{5}=\frac{\lambda^{3} \mu_{p}}{2 \pi} \int d^{p+1} \sigma & \sum_{p, n, m=0}^{\infty} e_{p, n, m}\left(\alpha^{\prime}\right)^{2 n+m}\left(\frac{\alpha^{\prime}}{2}\right)^{p} \times \\
\times & \operatorname{Tr}\left(C_{p-3} \wedge D^{b_{1}} \cdots D^{b_{m}} D^{a_{1}} \cdots D^{a_{2 n}} F \wedge\right. \\
& \left.\left(D^{a} D_{a}\right)^{p} D_{b_{1}} \cdots D_{b_{m}}\left[D_{a_{1}} \cdots D_{a_{n}} D \phi^{i} \wedge D_{a_{n+1}} \cdots D_{a_{2 n}} D \phi_{i}\right]\right)
\end{aligned}
$$

\section{Infinite massless gauge poles for $p=n$ case}

The goal for this section is to show that pure super Yang-Mills (SYM) couplings (infinite two gauge and two scalar couplings in [29]) will give rise the same infinite gauge poles in $<V_{C} V_{A} V_{\phi} V_{\phi}>$ as well. Extracting the trace and considring $L_{1}^{\prime}$ expansion inside of 
the third part of the amplitude $\left(A_{3}\right)$, we rewrite all infinite massless gauge poles of the amplitude for $p=n$ case as the following:

$$
\begin{aligned}
\mathcal{A}_{3}= & \pi^{3} \mu_{p} \xi_{3} \cdot \xi_{2} \frac{16}{p !} \epsilon^{a_{0} \cdots a_{p-1} a} H_{a_{0} \cdots a_{p-1}}^{(p)}\left[t s \xi_{1 a}-2 t k_{3} \cdot \xi_{1} k_{2 a}-2 s k_{2} \cdot \xi_{1} k_{3 a}\right] \\
& \times\left(\frac{\sum_{n, m=0}^{\infty} c_{n, m}\left[s^{n} t^{m}+s^{m} t^{n}\right]}{(t+s+u)}\right)
\end{aligned}
$$

with $2^{1 / 2} \pi^{1 / 2} \mu_{p}$ becomes normalisation factor to match with field theory side. The following Feynman rule must be taken into account for this case.

$$
\mathcal{A}=V_{\alpha}^{a}\left(C_{p-1}, A\right) G_{\alpha \beta}^{a b}(A) V_{\beta}^{b}\left(A, A_{1}, \phi_{2}, \phi_{3}\right),
$$

First of all let us talk about the chern -simons coupling, namely we want to gain $V_{\alpha}^{a}\left(C_{p-1}, A\right)$ by taking the known coupling

$$
2 \pi \alpha^{\prime} \mu_{p} \int d^{p+1} \sigma \operatorname{Tr}\left(C_{p-1} \wedge F\right)
$$

such that

$$
\begin{aligned}
V_{\alpha}^{a}\left(C_{p-1}, A\right) & =i\left(2 \pi \alpha^{\prime}\right) \mu_{p} \frac{1}{(p) !} \epsilon^{a_{0} \cdots a_{p-1} a} H_{a_{0} \cdots a_{p-1}}^{(p)} \operatorname{Tr}\left(\lambda_{\alpha}\right) \\
G_{\alpha \beta}^{a b}(A) & =\frac{i \delta_{\alpha \beta} \delta^{a b}}{T_{p}\left(2 \pi \alpha^{\prime}\right)^{2} k^{2}}=\frac{i \delta_{\alpha \beta} \delta^{a b}}{T_{p}\left(2 \pi \alpha^{\prime}\right)^{2}(t+s+u)} .
\end{aligned}
$$

In order to produce all infinite gauge poles for this particular case one has to know SYM couplings between one off-shell gauge and one on-shell gauge and two on-shell scalar fields at leading order

$$
-\frac{T_{p}\left(2 \pi \alpha^{\prime}\right)^{4}}{2} \operatorname{STr}\left(D_{a} \phi^{i} D^{b} \phi_{i} F^{a c} F_{b c}-\frac{1}{4}\left(D_{a} \phi^{i} D^{a} \phi_{i} F^{b c} F_{b c}\right)\right) .
$$

and in particular, we need to make use of their higher derivative corrections to all orders of $\alpha^{\prime}$ which are recently discovered in [29]:

$$
\begin{gathered}
\left(2 \pi \alpha^{\prime}\right)^{4} \frac{1}{2 \pi^{2}} T_{p}\left(\alpha^{\prime}\right)^{n+m} \sum_{m, n=0}^{\infty}\left(\mathcal{L}_{1}^{n m}+\mathcal{L}_{2}^{n m}+\mathcal{L}_{3}^{n m}\right), \\
\mathcal{L}_{1}^{n m}=-\operatorname{Tr}\left(a_{n, m} \mathcal{D}_{n m}\left[D_{a} \phi^{i} D^{b} \phi_{i} F^{a c} F_{b c}\right]+b_{n, m} \mathcal{D}_{n m}^{\prime}\left[D_{a} \phi^{i} F^{a c} D^{b} \phi_{i} F_{b c}\right]+\text { h.c. }\right), \\
\mathcal{L}_{2}^{n m}=-\operatorname{Tr}\left(a_{n, m} \mathcal{D}_{n m}\left[D_{a} \phi^{i} D^{b} \phi_{i} F_{b c} F^{a c}\right]+b_{n, m} \mathcal{D}_{n m}^{\prime}\left[D_{a} \phi^{i} F_{b c} D^{b} \phi_{i} F^{a c}\right]+\text { h.c. }\right), \\
\mathcal{L}_{3}^{n m}=\frac{1}{2} \operatorname{Tr}\left(a_{n, m} \mathcal{D}_{n m}\left[D_{a} \phi^{i} D^{a} \phi_{i} F^{b c} F_{b c}\right]+b_{n, m} \mathcal{D}_{n m}^{\prime}\left[D_{a} \phi^{i} F_{b c} D^{a} \phi_{i} F^{b c}\right]+\text { h.c. }\right),
\end{gathered}
$$

where the higher derivative operators $D_{n m}$ and $D_{n m}^{\prime}$ are defined [15] as

$$
\begin{aligned}
& \mathcal{D}_{n m}(E F G H) \equiv D_{b_{1}} \cdots D_{b_{m}} D_{a_{1}} \cdots D_{a_{n}} E F D^{a_{1}} \cdots D^{a_{n}} G D^{b_{1}} \cdots D^{b_{m}} H, \\
& \mathcal{D}_{n m}^{\prime}(E F G H) \equiv D_{b_{1}} \cdots D_{b_{m}} D_{a_{1}} \cdots D_{a_{n}} E D^{a_{1}} \cdots D^{a_{n}} F G D^{b_{1}} \cdots D^{b_{m}} H .
\end{aligned}
$$


The first thing to note is that, in order to obtain the vertex of one off-shell gauge and one gauge and two scalars on-shell, one should have taken into account two possible orderings as below:

$$
\operatorname{Tr}\left(\lambda_{2} \lambda_{3} \lambda_{1} \lambda_{\beta}\right), \quad \operatorname{Tr}\left(\lambda_{2} \lambda_{3} \lambda_{\beta} \lambda_{1}\right)
$$

where $\beta$ has to be Abelian. As an example if we consider $\operatorname{Tr}\left(a_{n, m} \mathcal{D}_{n m}\left[D_{a} \phi^{i} D^{b} \phi_{i} F^{a c} F_{b c}\right]\right)$ the resulted vertex is

$$
\begin{aligned}
a_{n, m}\left(k \cdot k_{2}\right)^{m}( & \left.k_{1} \cdot k_{2}\right)^{n} \xi_{2} \cdot \xi_{3} I_{10} \\
& +a_{n, m}\left(k \cdot k_{2}\right)^{n}\left(k_{1} \cdot k_{2}\right)^{m} \xi_{2} \cdot \xi_{3} I_{11}
\end{aligned}
$$

where $k$ becomes off-shell gauge field's momentum and $I_{10}, I_{11}$ are

$$
\begin{aligned}
& I_{10}=\left(-k_{1} \cdot k_{2} k_{3} \cdot k \xi_{1 a}+k_{1} \cdot k_{2} \xi_{1} \cdot k k_{3 a}+\xi_{1} \cdot k_{2} k_{3} \cdot k k_{1 a}-k_{2} \cdot \xi_{1} k_{1} \cdot k k_{3 a}\right) \\
& I_{11}=\left(-k \cdot k_{2} k_{3} \cdot k_{1} \xi_{1 a}+k \cdot k_{2} \xi_{1} \cdot k_{3} k_{1 a}+\xi_{1} \cdot k k_{3} \cdot k_{1} k_{2 a}-k_{3} \cdot \xi_{1} k_{1} \cdot k k_{2 a}\right)
\end{aligned}
$$

Now by applying the hermition conjugate of $\operatorname{Tr}\left(a_{n, m} \mathcal{D}_{n m}\left[D_{a} \phi^{i} D^{b} \phi_{i} F^{a c} F_{b c}\right]\right)$ we are lead to

$$
a_{n, m} \xi_{2} \cdot \xi_{3}\left(\left(k \cdot k_{3}\right)^{n}\left(k_{1} \cdot k_{3}\right)^{m} I_{10}+\left(k \cdot k_{3}\right)^{m}\left(k_{1} \cdot k_{3}\right)^{n} I_{11}\right)
$$

Therefore one must do careful computations for all the other couplings in (4.6) and also should consider their hermition conjugate as well. The final result is

$$
\begin{aligned}
V_{\beta}^{b}\left(A, \phi_{2}, \phi_{3}, A_{1}\right)= & \frac{T_{p}}{2} \xi_{2} \cdot \xi_{3} \frac{1}{2 \pi^{2}}\left(\alpha^{\prime}\right)^{n+m}\left(a_{n, m}+b_{n, m}\right)\left(\left(k_{2} \cdot k\right)^{m}\left(k_{1} \cdot k_{2}\right)^{n}+\left(k_{2} \cdot k\right)^{n}\left(k_{2} \cdot k_{1}\right)^{m}\right. \\
& \left.+\left(k_{1} \cdot k_{3}\right)^{m}\left(k_{3} \cdot k\right)^{n}+\left(k \cdot k_{3}\right)^{m}\left(k_{1} \cdot k_{3}\right)^{n}\right)\left(2 \pi \alpha^{\prime}\right)^{4} \operatorname{Tr}\left(\lambda_{1} \lambda_{2} \lambda_{3} \lambda_{\beta}\right) \\
& \times\left[\xi_{1 b} t s-2 t k_{3} \cdot \xi_{1} k_{2 b}-2 s k_{2} \cdot \xi_{1} k_{3 b}\right],
\end{aligned}
$$

Having set (4.11),(4.4) into (4.2), we get the infinite massless gauge field poles of the amplitude in field theory side:

$$
\begin{array}{r}
-32 \pi \mu_{p} \frac{\epsilon^{a_{0} \cdots a_{p-1} a} \xi_{2} \cdot \xi_{3} H_{a_{0} \cdots a_{p-1}}^{(p)}}{(p) !(s+t+u)} \operatorname{Tr}\left(\lambda_{1} \lambda_{2} \lambda_{3}\right) \sum_{n, m=0}^{\infty}\left(a_{n, m}+b_{n, m}\right)\left[s^{m} t^{n}+s^{n} t^{m}\right] \\
{\left[\xi_{1 a} t s-2 t k_{3} \cdot \xi_{1} k_{2 a}-2 s k_{2} \cdot \xi_{1} k_{3 a}\right]}
\end{array}
$$

In order to check the field theory amplitude with string amplitude (4.1) one needs to actually have some of the coefficients such as

$$
\begin{array}{ll}
a_{0,0}=-\frac{\pi^{2}}{6}, & b_{0,0}=-\frac{\pi^{2}}{12}, \\
a_{1,0}=2 \zeta(3), & a_{0,1}=0 \\
b_{0,1}=-\zeta(3), & a_{1,1}=a_{0,2}=-7 \pi^{4} / 90 \\
a_{2,2}=\left(-83 \pi^{6}-7560 \zeta(3)^{2}\right) / 945, & b_{2,2}=-\left(23 \pi^{6}-15120 \zeta(3)^{2}\right) / 1890
\end{array}
$$




$$
\begin{array}{llrl}
a_{1,3} & =-62 \pi^{6} / 945, & a_{2,0}=-4 \pi^{4} / 90, \\
b_{1,1} & =-\pi^{4} / 180, & b_{0,2}=-\pi^{4} / 45, \\
a_{0,4}=-31 \pi^{6} / 945, & a_{4,0}=-16 \pi^{6} / 945, \\
a_{1,2}=a_{2,1}=8 \zeta(5)+4 \pi^{2} \zeta(3) / 3, & a_{0,3}=0, \\
a_{3,0}=8 \zeta(5), & b_{1,3}=-\left(12 \pi^{6}-7560 \zeta(3)^{2}\right) / 1890, \\
a_{3,1}=\left(-52 \pi^{6}-7560 \zeta(3)^{2}\right) / 945, & b_{0,3}=-4 \zeta(5), \\
b_{1,2}=-8 \zeta(5)+2 \pi^{2} \zeta(3) / 3, & b_{0,4}=-16 \pi^{6} / 1890 .
\end{array}
$$

Notice the fact that $b_{n, m}$ must be symmetric and concerning T-duality transformation these coefficients are the same as those have been appeared for one RR and 3 gauge fields [15]. Later on we will go through all of the contact terms for $p=n$ case, even those terms which have been cancelled out with the resulted propagator in the above field theory amplitude. Although the method for obtaining them with all needed details have been explained in $[29,31]$.

Meanwhile the amplitude in string theory is given in (4.1). If the higher derivative couplings of (4.6) are correct, we must be able to produce exactly all massless poles in (4.1). To do so, first we omit similar coefficients from both string and field amplitudes and then compare (4.12) with (4.1) order by order. In the other words, the aim is to compare

$$
-\mu_{p} \pi \sum_{n, m=0}^{\infty}\left(\left(a_{n, m}+b_{n, m}\right)\left[s^{m} t^{n}+s^{n} t^{m}\right]\right)
$$

with

$$
2^{-1} \pi^{3} \mu_{p} \sum_{n, m=0}^{\infty} c_{n, m}\left(s^{m} t^{n}+s^{n} t^{m}\right)
$$

By applying $n=m=0$, at zeroth order of $\alpha^{\prime}$ we get

$$
-2 \pi\left(a_{0,0}+b_{0,0}\right)=-2 \pi\left(\frac{-\pi^{2}}{6}+\frac{-\pi^{2}}{12}\right)=\frac{\pi^{3}}{2}\left(2 c_{0,0}\right)
$$

At first order of $\alpha^{\prime}$, we find

$$
-\pi\left(a_{1,0}+a_{0,1}+b_{1,0}+b_{0,1}\right)(s+t)=0=\frac{\pi^{3}}{2}\left(c_{1,0}+c_{0,1}\right)(s+t)
$$

At the second order of $\left(\alpha^{\prime}\right)$, we lead to

$$
\begin{aligned}
-2 \pi\left(a_{1,1}\right. & \left.+b_{1,1}\right) s t-\pi\left(a_{0,2}+a_{2,0}+b_{0,2}+b_{2,0}\right)\left[s^{2}+t^{2}\right] \\
& =\frac{\pi^{5}}{6}(s t)+\frac{\pi^{5}}{6}\left(s^{2}+t^{2}\right) \\
& =\frac{\pi^{3}}{2}\left[c_{1,1}(2 s t)+\left(c_{2,0}+c_{0,2}\right)\left(s^{2}+t^{2}\right)\right]
\end{aligned}
$$

At third order of $\alpha^{\prime}$, we gain

$$
\begin{aligned}
-\pi\left(a_{3,0}\right. & \left.+a_{0,3}+b_{0,3}+b_{3,0}\right)\left[s^{3}+t^{3}\right]-\pi\left(a_{1,2}+a_{2,1}+b_{1,2}+b_{2,1}\right)[s t(s+t)] \\
& =-4 \pi^{3} \xi(3) s t(s+t)=\frac{\pi^{3}}{2}\left[\left(c_{0,3}+c_{3,0}\right)\left[s^{3}+t^{3}\right]+\left(c_{2,1}+c_{1,2}\right) s t(s+t)\right]
\end{aligned}
$$


In order to be sure we have obtained the correct couplings with exact coefficients, we want to go ahead one more order so at fourth order of $\left(\alpha^{\prime}\right)$, we find the following numerical factor

$$
\begin{aligned}
-\pi\left(a_{4,0}+a_{0,4}+b_{0,4}+b_{4,0}\right)\left(s^{4}+t^{4}\right) \\
-\pi\left(a_{3,1}+a_{1,3}+b_{3,1}+b_{1,3}\right)\left[s t\left(s^{2}+t^{2}\right)\right]-2 \pi\left(a_{2,2}+b_{2,2}\right) s^{2} t^{2} \\
=\frac{\pi^{7}}{15}\left(s^{4}+t^{4}+2\left(s^{3} t+t^{3} s\right)+3 s^{2} t^{2}\right) \\
=\frac{\pi^{3}}{2}\left[\left(c_{4,0}+c_{0,4}\right)\left(s^{4}+t^{4}\right)+\left(c_{1,3}+c_{3,1}\right)\left(s^{3} t+t^{3} s\right)+2 c_{2,2} s^{2} t^{2}\right]
\end{aligned}
$$

We have highly used the coefficients in (4.13). In general all checks to all orders in $\alpha^{\prime}$ can be carried out to indeed see that all massless gauge poles of $\left\langle V_{C} V_{A} V_{\phi} V_{\phi}>\right.$ are produced. Therefore we come to important fact that these couplings do work out even for the amplitude of $C A \phi \phi$, and this is the other recent point that comes out from our attempts which has been hidden in [55] for a while. Therefore not only does it confirm that our recent higher derivative couplings are exact up to on-shell ambiguity but also it resolves the fact that $p_{a} p^{a}$ must tend to zero to get the correct expansion for all BPS branes.

\subsection{Infinite massless $t, s$-channel scalar poles for $p+2=n$ case}

The goal in this section is to actually produce all infinite s-channel and t-channel scalar poles. The first simple scalar pole in t-channel has already been produced in [55] but again in there all infinite scalar poles have been overlooked, however we are going to come over them as well.

Having taken our recent ideas for Super Yang-Mills [15, 29, 30], we show that the same arguments here also hold. By applying $u s L_{2}^{\prime}, t u L_{2}^{\prime}$ expansions into all terms (except the second term) in the $\mathcal{A}_{2}$ amplitude and extracting the traces, one can find out all massless scalar poles in t channel for string amplitude as

$$
\begin{aligned}
\frac{-16 \pi^{2} \mu_{p}}{(p+1) !}\{ & \left.-2(p+1) \xi_{1} \cdot k_{2} \xi_{2 i} k_{3 c} \xi_{3 j} \epsilon^{a_{0} \cdots a_{p-1} c} H_{a_{0} \cdots a_{p-1}}^{i j}+2 \xi_{2 i} p^{j} \xi_{1} \cdot k_{2} \xi_{3 j} \epsilon^{a_{0} \cdots a_{p}} H_{a_{0} \cdots a_{p}}^{i}\right\} \\
& \times \sum_{n=-1}^{\infty} b_{n}\left(\frac{1}{t}(u+s)^{n+1}\right) \operatorname{Tr}\left(\lambda_{1} \lambda_{2} \lambda_{3}\right)
\end{aligned}
$$

All s-channel poles are also written down as below

$$
\begin{gathered}
\frac{-16 \pi^{2} \mu_{p}}{(p+1) !}\left\{2(p+1) k_{3} \cdot \xi_{1} k_{2 b} \xi_{3 j} \xi_{2 i} \epsilon^{a_{0} \cdots a_{p-1} b} H_{a_{0} \cdots a_{p-1}}^{j i}-2 \xi_{3 j} p^{i} \xi_{1} \cdot k_{3} \xi_{2 i} \epsilon^{a_{0} \cdots a_{p}} H_{a_{0} \cdots a_{p}}^{j}\right\} \\
\times \sum_{n=-1}^{\infty} b_{n}\left(\frac{1}{s}(u+t)^{n+1}\right) \operatorname{Tr}\left(\lambda_{1} \lambda_{2} \lambda_{3}\right)
\end{gathered}
$$

By interchanging scalars in the $\mathcal{A}_{2}$ amplitude, we reach to the point that the amplitude is anti symmetric thus in order to make sense of our computations one has to consider nonAbelian gauge group. As it is clear from (4.18) and (4.17), once we produced all massless t-channel scalar poles, all infinite s-channel scalar poles can be easily produced by replacing 
$s \leftrightarrow t$ and re-labeling $2 \leftrightarrow 3$ in all their momenta and polarizations. Therefore let us just produce all infinite massless scalar poles in t-channel in field theory.

The Feynman rule in field theory to produce all t-channel poles should be followed by

$$
\mathcal{A}=V_{\alpha}^{i}\left(C_{p+1}, \phi_{3}, \phi\right) G_{\alpha \beta}^{i j}(\phi) V_{\beta}^{j}\left(\phi, A_{1}, \phi_{2}\right),
$$

such that the vertex of $V_{\beta}^{j}\left(\phi, A_{1}, \phi_{2}\right)$ should be found from the scalar field's kinetic term like $\frac{\left(2 \pi \alpha^{\prime}\right)^{2}}{2} \operatorname{Tr}\left(D_{a} \phi^{i} D^{a} \phi_{i}\right)$ where all possible orderings must be regarded in field theory as well. Therefore

$$
\begin{aligned}
V_{\beta}^{j}\left(\phi, A_{1}, \phi_{2}\right) & =-2 i \lambda^{2} T_{p} k_{2} \cdot \xi_{1} \xi_{2}^{j} \operatorname{Tr}\left(\lambda_{1} \lambda_{2} \lambda_{\beta}\right) \\
G_{\alpha \beta}^{i j}(\phi) & =\frac{-i}{N \lambda^{2} T_{p}} \frac{\delta^{i j} \delta_{\alpha \beta}}{k^{2}}
\end{aligned}
$$

$k^{2}=-\left(k_{2}+k_{1}\right)^{2}=t$ should be substituted in the propagator. As argued in the last section scalar field 's kinetic term has been fixed so it has no correction, the simple scalar t-channel pole has no correction either. Therefore not only we need to find $V_{\alpha}^{i}\left(C_{p+1}, \phi_{3}, \phi\right)$ but also its higher derivative corrections are also needed.

First of all let us discuss how to produce $V_{\alpha}^{i}\left(C_{p+1}, \phi_{3}, \phi\right)$ without taking its higher derivative.

The first coupling between one gauge field, two scalar fields and one RR should be included from Myers ' terms. Namely, we may think of the coupling between a commutator of transverse scalars and a world volume field strength of gauge field and one RR - $(p+1)$ form field as we call it $S_{6}$

$$
S_{6}=\frac{i}{4}\left(2 \pi \alpha^{\prime}\right)^{2} \mu_{p} \int d^{p+1} \sigma \frac{1}{(p-1) !} \epsilon^{a_{0} \cdots a_{p}} \operatorname{Tr}\left(F_{a_{0} a_{1}}\left[\Phi^{j}, \Phi^{i}\right]\right) C_{i j a_{2} \cdots a_{p}}^{(p+1)} .
$$

For more details on Chern-Simons actions, Taylor expansion and Pull-back, one should deal with section 5 of [31]. In addition to (4.21) we need to know two more couplings, basically first we need to use Taylor expansion very properly for this case as

$$
\begin{aligned}
S_{7} & =\frac{\left(2 \pi \alpha^{\prime}\right)^{2} \mu_{p}}{2} \int d^{p+1} \sigma \frac{1}{(p+1) !} \epsilon^{a_{0} \cdots a_{p}} \operatorname{Tr}\left(\Phi^{j} \Phi^{i}\right) \partial_{j} \partial_{i} C_{a_{0} \cdots a_{p}}^{(p+1)} \\
& =\frac{\left(2 \pi \alpha^{\prime}\right)^{2} \mu_{p}}{2} \int d^{p+1} \sigma \frac{1}{(p+1) !} \epsilon^{a_{0} \cdots a_{p}} \operatorname{Tr}\left(\Phi^{j} \Phi^{i}\right) \partial_{j} H_{i a_{0} \cdots a_{p}}^{(p+2)}
\end{aligned}
$$

such that $H^{p+2}=d C^{p+1}$, the other couplings which are vital for our case must be read from Pull-back, namely we shall point out to the following couplings as well

$$
\begin{aligned}
S_{8}=\frac{\left(2 \pi \alpha^{\prime}\right)^{2} \mu_{p}}{2} \int d^{p+1} \sigma \frac{1}{(p+1) !} \epsilon^{a_{0} \cdots a_{p}}\left[p(p+1) \operatorname{Tr}\left(D_{a_{0}} \Phi^{i} D_{a_{1}} \Phi^{j}\right) C_{i j a_{2} \cdots a_{p}}^{(p+1)}\right. \\
\left.+2(p+1) \operatorname{Tr}\left(\Phi^{j} D_{a_{0}} \Phi^{i}\right) \partial_{j} C_{i a_{1} \cdots a_{p}}^{(p+1)}\right]
\end{aligned}
$$

Having taken integration by parts and adding some of the actions we reach to

$$
S_{6}+S_{8}=\frac{\left(2 \pi \alpha^{\prime}\right)^{2}}{2} \mu_{p} \int d^{p+1} \sigma \frac{1}{(p+1) !} \epsilon^{a_{0} \cdots a_{p}}\left[(p+1) \operatorname{Tr}\left(D_{a_{0}} \Phi^{j} \Phi^{i}\right) H_{i j a_{1} \cdots a_{p}}^{(p+2)}\right]
$$


In order to get several contributions, one has to extract the covariant derivative of scalar field $\left(D_{a} \phi^{i}=\partial_{a} \phi^{i}+i\left[A_{a}, \phi^{i}\right]\right)$ such that

$$
\begin{aligned}
& S_{6}+S_{8}=\frac{\left(2 \pi \alpha^{\prime}\right)^{2}}{2} \mu_{p} \int d^{p+1} \sigma \frac{1}{(p+1) !} \epsilon^{a_{0} \cdots a_{p}}\left[2 i(p+1) \operatorname{Tr}\left(A_{a_{0}} \Phi^{j} \Phi^{i}\right) H_{i j a_{1} \cdots a_{p}}^{(p+2)}\right. \\
& \left.+(p+1) \operatorname{Tr}\left(\partial_{a_{0}} \Phi^{j} \Phi^{i}\right) H_{i j a_{1} \cdots a_{p}}^{(p+2)}\right]
\end{aligned}
$$

Note that the first term in (4.23) will be employed in the next section to obtain all the contact terms $p+2=n$ case for three open strings, namely two scalars, one gauge field and one closed string RR- $p+1$ form field.

However, in order to obtain $V_{\alpha}^{i}\left(C_{p+1}, \phi_{3}, \phi\right)$ for producing the first massless scalar pole,one must add the relevant couplings together at leading order, basically by adding some of the couplings as below

$$
\frac{\mu_{p}\left(2 \pi \alpha^{\prime}\right)^{2}}{2(p+1) !} \int d^{p+1} \sigma \epsilon^{a_{0} \cdots a_{p}}\left[\operatorname{Tr}\left(\Phi^{j} \Phi^{i}\right) \partial_{j} H_{i a_{0} \cdots a_{p}}^{(p+2)}+(p+1) \operatorname{Tr}\left(\partial_{a_{0}} \Phi^{j} \Phi^{i}\right) H_{i j a_{1} \cdots a_{p}}^{(p+2)}\right]
$$

we can easily get the leading vertex of $V_{\alpha}^{i}\left(C_{p+1}, \phi_{3}, \phi\right)$ as

$$
\begin{aligned}
V_{\alpha}^{i}\left(C_{p+1}, \phi_{3}, \phi\right)=\frac{N \mu_{p}\left(2 \pi \alpha^{\prime}\right)^{2}}{(p+1) !} \operatorname{Tr}\left(\lambda_{3} \lambda_{\alpha}\right) \epsilon^{a_{0} \cdots a_{p}}[ & p^{j} \xi_{3 j} H_{a_{0} \cdots a_{p}}^{i} \\
& \left.+(p+1) H_{a_{1} \cdots a_{p}}^{i j} k_{3 a_{0}} \xi_{3 j}\right]
\end{aligned}
$$

Needless to say that $N$ is indeed the normalisation constant to be chosen for all $\mathrm{U}(\mathrm{N})$ generators, such that

$$
\xi_{1 i}=\xi_{1 i}^{\alpha} Q_{\alpha}, \quad N \delta^{\alpha \beta}=\operatorname{Tr}\left(Q^{\alpha} Q^{\beta}\right)
$$

Now by replacing (4.25) and (4.20) into (4.19), we are able to just produce exactly the first simple t-channel pole in (4.17). In order to produce all infinite t-channel poles, we should look for all higher derivative corrections of (4.24). One can apply the main ideas of $[15,29]$ to indeed get the all higher derivative corrections of (4.24) as

$$
\begin{gathered}
\frac{\mu_{p}\left(2 \pi \alpha^{\prime}\right)^{2}}{2(p+1) !} \int d^{p+1} \sigma \epsilon^{a_{0} \cdots a_{p}} \sum_{n=-1}^{\infty} b_{n}\left(\alpha^{\prime}\right)^{n}\left[\operatorname{Tr}\left(D_{a_{1} \ldots a_{n}} \Phi^{j} D^{a_{1} \ldots a_{n}} \Phi^{i}\right) \partial_{j} H_{i a_{0} \cdots a_{p}}^{(p+2)}\right. \\
\left.+(p+1) \operatorname{Tr}\left(\partial_{a_{0}} D_{a_{1} \ldots a_{n}} \Phi^{j} D^{a_{1} \ldots a_{n}} \Phi^{i}\right) H_{i j a_{1} \cdots a_{p}}^{(p+2)}\right]
\end{gathered}
$$

The important point here is that the commutators in covariant derivative of scalar fields do not play any role and in fact they have no contribution to above vertex so all covariant derivatives can be replaced with their own partial derivatives.

By constructing the correct higher derivative corrections of (4.24) as (4.27), one can write down the general form of the needed vertex as

$$
\begin{aligned}
V_{\alpha}^{i}\left(C_{p+1}, \phi_{3}, \phi\right)=\frac{N \mu_{p}\left(2 \pi \alpha^{\prime}\right)^{2}}{(p+1) !} \operatorname{Tr}\left(\lambda_{3} \lambda_{\alpha}\right) \epsilon^{a_{0} \cdots a_{p}} \sum_{n=-1}^{\infty} b_{n}\left(\alpha^{\prime} k_{3} \cdot k\right)^{n}\left[p^{j} \xi_{3 j} H_{a_{0} \cdots a_{p}}^{i}\right. \\
\left.+(p+1) H_{a_{1} \cdots a_{p}}^{i j} k_{3 a_{0}} \xi_{3 j}\right]
\end{aligned}
$$

where $\sum_{n=-1}^{\infty} b_{n}\left(\alpha^{\prime} k_{3} \cdot k\right)^{n}=\sum_{n=-1}^{\infty} b_{n}(s+u)^{n}$ has been used. 
Therefore by making use of this new higher vertex (to all orders of $\alpha^{\prime}$ ) (4.28) and substituting (4.28) and (4.20) into (4.19), fortunately we were able to exactly obtain all infinite t-channel scalar poles in (4.17) as follows

$$
\begin{aligned}
\mathcal{A}= & \frac{-16 \pi^{2} \mu_{p}}{(p+1) !}\left\{-2(p+1) \xi_{1} \cdot k_{2} \xi_{2 i} k_{3 a} \xi_{3 j} \epsilon^{a_{0} \cdots a_{p-1} a} H_{a_{0} \cdots a_{p-1}}^{i j}\right. \\
& \left.+2 \xi_{2 i} p^{j} \xi_{1} \cdot k_{2} \xi_{3 j} \epsilon^{a_{0} \cdots a_{p}} H_{a_{0} \cdots a_{p}}^{i}\right\} \\
& \times \sum_{n=-1}^{\infty} b_{n}\left(\frac{1}{t}(u+s)^{n+1}\right) \operatorname{Tr}\left(\lambda_{1} \lambda_{2} \lambda_{3}\right)
\end{aligned}
$$

Having replaced $t \leftrightarrow s$ and $2 \leftrightarrow 3$ we can also produce all infinite s-channel scalar poles as well.

Thus up to pole levels we observe that field theory does agree with string amplitude, however in the next sections we will see that there are some contact terms in string theory such that their field theory is unknown. It is remarkable to note that these sort of new interactions neither can be found by Myers'terms nor with Taylor/Pull back method. To our knowledge pull-back should be corrected [29]. Essentially we find some new couplings by comparing them with the exact result of string amplitude. After carrying out long computations and producing all infinite massless scalar, gauge poles for all possible different channels, let us go further and talk about contact interactions and new Wess-Zumino couplings, which can be found just by direct S-Matrix computations.

\section{Contact interactions for $p=n$ case}

Notice the fact that $p=n$ does mean that we are taking into account all $C_{p-1}$ couplings to $D_{(p-2)}$-brane. By taking $n=p$ case, the final form of our amplitude reduced to the following interactions:

$$
\begin{aligned}
& A^{C A \phi \phi}=\frac{i\left(2 \pi \alpha^{\prime}\right)^{3} \mu_{p}}{2 p !} \epsilon^{a_{0} \cdots a_{p-1} a} \xi_{1 a} \xi_{2 i} \xi_{3 j}\left(p^{i} p^{j} H_{a_{0} \cdots a_{p-1}}+p(p-1) k_{3 a_{0}} k_{2 a_{1}} H_{a_{2} \cdots a_{p-1}}^{i j}\right. \\
& \left.-p k_{2 a_{0}} p^{j} H_{a_{1} \cdots a_{p-1}}^{i}-p k_{3 a_{0}} p^{i} H_{a_{1} \cdots a_{p-1}}^{j}\right) \\
& \times\left(\sum_{n=0}^{\infty} c_{n}(s+t+u)^{n+1}+\sum_{n, m=0}^{\infty} c_{n, m}\left[(s)^{n}(t)^{m}+(s)^{m}(t)^{n}\right]\right. \\
& \left.+\sum_{p, n, m=0}^{\infty} f_{p, n, m}(s+t+u)^{p+1}\left[(s+t)^{n}(s t)^{m}\right]\right) \text {, }
\end{aligned}
$$

Some of the contact terms just at the leading order for this case were known in [55]. Note that we are just considering $\operatorname{Tr}\left(\lambda_{1} \lambda_{2} \lambda_{3}\right)$ while the amplitude has the other possible ordering which is $\operatorname{Tr}\left(\lambda_{1} \lambda_{3} \lambda_{2}\right)$. In order to obtain full amplitude one has to replace $s \leftrightarrow u$ and $2 \leftrightarrow 3$ in the above contact interactions and add them up with (5.1). In order to produce (5.1) we have to consider several couplings from field theory. Let us first reconsider the couplings 
between two scalars (coming from pull-back) and one gauge field as

$$
\begin{array}{r}
S_{9}=\frac{\left(2 \pi \alpha^{\prime}\right)^{3} \mu_{p}}{4(p-1) !} \int d^{p+1} \sigma \epsilon^{a_{0} \cdots a_{p}}\left((p-2)(p-1) \operatorname{S} \operatorname{Tr}\left(F_{a_{0} a_{1}} D_{a_{2}} \Phi^{i} D_{a_{3}} \Phi^{j}\right) C_{i j a_{4} \cdots a_{p}}\right. \\
\left.+2(p-1) \operatorname{S} \operatorname{Tr}\left(F_{a_{0} a_{1}} D_{a_{2}} \Phi^{i} \Phi^{j}\right) \partial_{j} C_{i a_{3} \cdots a_{p}}\right)
\end{array}
$$

The important point for the above coupling is that commutator in the definition of covariant derivative of scalars must be overlooked as we are looking for two scalars and one gauge coupling. As a matter of fact all covariant derivative should be replaced with their partial derivative.

The other coupling (which is essential for producing all contact terms for $p=n$ case), should be coming from Taylor expansions as follows

$$
S_{10}=\frac{\left(2 \pi \alpha^{\prime}\right)^{3} \mu_{p}}{4(p-1) !} \int d^{p+1} \sigma \epsilon^{a_{0} \cdots a_{p}} \operatorname{STr}\left(F_{a_{0} a_{1}} \Phi^{i} \Phi^{j}\right) \partial_{i} \partial_{j} C_{a_{2} \cdots a_{p}}
$$

Now by extracting field strength, replacing all covariant derivatives with their partial derivatives, adding (5.3) and (5.2) $\left(S_{11}=S_{9}+S_{10}\right)$ and finally replacing all fields to their polarizations and in particular changing derivatives to momenta, we can precisely produce all contact terms at the leading order with the following couplings:

$$
\begin{aligned}
S_{11}=\frac{\lambda^{3} \mu_{p}}{2 p !} \int & d^{p+1} \sigma \epsilon^{a_{0} \cdots a_{p}}\left(p(p-1) \operatorname{STr}\left(A_{a_{0}} \partial_{a_{1}} \Phi^{i} \partial_{a_{2}} \Phi^{j}\right) H_{i j a_{3} \cdots a_{p}}\right. \\
& \left.+2 p \operatorname{STr}\left(A_{a_{0}} \partial_{a_{1}} \Phi^{j} \Phi^{i}\right) \partial_{i} H_{j a_{2} \cdots a_{p}}+\operatorname{STr}\left(A_{a_{0}} \Phi^{i} \Phi^{j}\right) \partial_{i} \partial_{j} H_{a_{1} \cdots a_{p}}\right)
\end{aligned}
$$

Note that the first term in (5.4) is derived from (5.2) where all the commutators should be dropped, as we are looking for the couplings between two scalars and one gauge and one RR $-p-1$ form field.

Also note that, in the first term of (5.2), the partial derivative inside the field strength can not act on scalars because the $\epsilon$ tensor is antisymmetric and the multiplication of symmetric tensor and antisymmetrc tensor becomes zero so it can act just on RR field. Symmetric trace does mean that, taking average on the whole possible orderings of the fields is vital. The appearance of symmetric trace for the last term is necessary as we have to produce the third and the last term in (5.1) very precisely.

In order to produce all infinite contact interactions in (5.1), the following higher derivative corrections should have been taken into account.

$$
\begin{aligned}
(s t)^{m} H A \phi \phi & =\left(\alpha^{\prime}\right)^{2 m} H \partial_{a_{1}} \cdots \partial_{a_{2 m}} A D^{a_{1}} \cdots D^{a_{m}} \phi D^{a_{m+1}} \cdots D^{a_{2 m}} \phi \\
(s+t)^{n} H A \phi \phi & =\left(\alpha^{\prime}\right)^{n} H \partial_{a_{1}} \cdots \partial_{a_{n}} A D^{a_{1}} \cdots D^{a_{n}}(\phi \phi), \\
(s)^{m} t^{n} H A \phi \phi & =\left(\alpha^{\prime}\right)^{n+m} H \partial_{a_{1}} \cdots \partial_{a_{n}} \partial_{a_{1}} \cdots \partial_{a_{m}} A D^{a_{1}} \cdots D^{a_{n}} \phi D^{a_{1}} \cdots D^{a_{m}} \phi \\
(s)^{n} t^{m} H A \phi \phi & =\left(\alpha^{\prime}\right)^{n+m} H \partial_{a_{1}} \cdots \partial_{a_{n}} \partial_{a_{1}} \cdots \partial_{a_{m}} A D^{a_{1}} \cdots D^{a_{m}} \phi D^{a_{1}} \cdots D^{a_{n}} \phi \\
(s+t+u)^{p+1} H A \phi \phi & =\left(\frac{\alpha^{\prime}}{2}\right)^{p+1} H\left(D_{a} D^{a}\right)^{p+1}(A \phi \phi) .
\end{aligned}
$$


The important point which must be highlighted is that, the connection part or the commutator in the covariant derivative of scalars must be dropped in the above couplings.

\section{New couplings for $p=n$ case}

Carrying out the trace and considering only all related contact interactions for $A_{3}$, we get the terms like

$$
\begin{aligned}
A_{3}^{C A \phi \phi}= & -\frac{16 \pi^{3} \mu_{p}}{(p) !} \xi_{3} \cdot \xi_{2} H_{a_{0} \cdots a_{p-1}} \epsilon^{a_{0} \cdots a_{p-1} a}\left[\frac{t s}{2} \xi_{1 a}-2 t k_{3} \cdot \xi_{1} k_{2 a}+2 \leftrightarrow 3\right] \\
& \times\left(\sum_{n=0}^{\infty} c_{n}(s+t+u)^{n}+\sum_{p, n, m=0}^{\infty} f_{p, n, m}(s+t+u)^{p}(s t)^{m}(s+t)^{n}\right)
\end{aligned}
$$

These new contact terms do satisfy related Ward identity. It is really worth trying to point out that these contact terms are sort of new couplings. In principle we should be able to produce these new couplings by introducing new couplings in field theory.

Keep in mind that, we proceed to find out new couplings in field theory term by term however after all, one has to add all of them together. Let us first produce the first term in (6.1) at leading order (remember $c_{1}=\frac{\pi^{2}}{6}, f_{0,1,0}=\frac{-\pi^{2}}{3}$ ) then we generalize its higher order corrections to all orders in $\alpha^{\prime}$, namely consider

$$
-\frac{16 \pi^{3} \mu_{p}}{(p) !} \xi_{3} \cdot \xi_{2} H_{a_{0} \cdots a_{p-1}} \epsilon^{a_{0} \cdots a_{p-1} a} \frac{t s}{2} \xi_{1 a}\left(\frac{\pi^{2}}{6}(s+t+u)-\frac{\pi^{2}}{3}(s+t)\right)
$$

One can exactly produce (6.2) by taking into account the fact that the sum of world volume indices must cover all world volume indices such that the first term in (6.2) is obtained by the new coupling as

$$
S_{12}=\frac{\left(2 \pi \alpha^{\prime}\right) \mu_{p}\left(\pi^{2} \alpha^{\prime}\right)^{2}}{3} \int d^{p+1} \sigma C_{(p-1)} \wedge\left(\frac{\alpha^{\prime}}{2}\left(D^{c} D_{c}\right)\left(D^{b} D^{a} F D_{a} \Phi^{i} D_{b} \Phi_{i}\right)\right)
$$

Now we can generalize the above coupling (6.3) to produce all non leading couplings for the first term in (6.2) as

$$
S_{13}=\frac{\left(2 \pi \alpha^{\prime}\right)^{3} \mu_{p}}{2} \int d^{p+1} \sigma \sum_{n=0}^{\infty} c_{n}\left(\frac{\alpha^{\prime}}{2}\right)^{n} C_{(p-1)} \wedge\left(D^{c} D_{c}\right)^{n}\left(D^{b} D^{a} F D_{a} \Phi^{i} D_{b} \Phi_{i}\right),
$$

In those couplings, we have written explicit covariant derivative of the scalar fields, however with our computations we can confirm the presence of just the partial derivatives in covariant derivatives, thus in order to check whether or not commutators should be held, one should perform higher point functions just like $C A A \phi \phi[57]$.

Pursuing the argument mentioned above, one can produce the second term in (6.2) as follows

$$
S_{14}=-\frac{\left(8 \pi \alpha^{\prime}\right) \mu_{p}\left(\alpha^{\prime} \pi^{2}\right)^{2}}{3} \int d^{p+1} \sigma C_{(p-1)} \wedge\left(D^{c} D^{a} D^{b} F D_{c}\left[D_{a} \Phi^{i} D_{b} \Phi_{i}\right]\right),
$$


Making use of the steps have been mentioned in [31], we are able to get the all higher order corrections for the second term in (6.2) as

$$
\begin{aligned}
S_{15}= & -\frac{\left(2 \pi \alpha^{\prime}\right)^{3} \mu_{p}}{2} \int d^{p+1} \sigma \sum_{p, n, m=0}^{\infty} f_{p, n, m}\left(\frac{\alpha^{\prime}}{2}\right)^{p}\left(\alpha^{\prime}\right)^{2 m+n} C_{(p-1)} \wedge\left(D^{a} D_{a}\right)^{p}\left(D_{b_{1}} \cdots D_{b_{2 m}}\right. \\
& \left.D^{a_{1}} \cdots D^{a_{n}} D^{b} D^{c} F D_{a_{1}} \cdots D_{a_{n}}\left[D^{b_{1}} \cdots D^{b_{m}} D_{b} \Phi^{i} D^{b_{m+1}} \cdots D^{b_{2 m}} D_{c} \Phi_{i}\right]\right),
\end{aligned}
$$

In order to produce the rest of the terms in (6.1), namely one has to consider the following terms

$$
\begin{aligned}
&-\frac{16 \pi^{3} \mu_{p}}{(p) !} \xi_{3} \cdot \xi_{2} H_{a_{0} \cdots a_{p-1}} \epsilon^{a_{0} \cdots a_{p-1} a}\left[-2 t k_{3} \cdot \xi_{1} k_{2 a}+2 \leftrightarrow 3\right] \\
& \times\left(\sum_{n=0}^{\infty} c_{n}(s+t+u)^{n}+\sum_{p, n, m=0}^{\infty} f_{p, n, m}(s+t+u)^{p}(s t)^{m}(s+t)^{n}\right)
\end{aligned}
$$

To produce the first term in (6.7) at leading order, one must consider the following coupling and adds it up to (6.3) and (6.5)

$$
S_{16}=\frac{\left(2 \pi \alpha^{\prime}\right)^{3} \mu_{p} \pi^{2} \epsilon^{a_{0} \cdots a_{p}} H_{a_{0} \cdots a_{p-1}} \alpha^{\prime}\left(D_{a} D^{a}\right)}{12 p !} \int d^{p+1} \sigma\left(\partial_{c} A_{b} \partial_{a_{p}} D^{c} \Phi_{i} D^{b} \Phi^{i}-2 \leftrightarrow 3\right)
$$

Now we can generalize the above coupling to produce all non leading couplings to all orders of $\alpha^{\prime}$

$$
\begin{aligned}
& S_{17}=\frac{\left(2 \pi \alpha^{\prime}\right)^{3} \mu_{p}}{p !} \epsilon^{a_{0} \cdots a_{p}} \int d^{p+1} \sigma H_{a_{0} \cdots a_{p-1}} \sum_{n=0}^{\infty} c_{n}\left(\frac{\alpha^{\prime}}{2}\right)^{n}\left(D_{a} D^{a}\right)^{n} \\
& \times\left(\partial_{c} A_{b} \partial_{a_{p}} D^{c} \Phi_{i} D^{b} \Phi^{i}-2 \leftrightarrow 3\right)
\end{aligned}
$$

Also notice that the second term in (6.7) at leading order can be reproduced as

$$
S_{18}=\frac{\left(2 \pi \alpha^{\prime}\right)^{3} \mu_{p} \pi^{2}}{3 p !} \epsilon^{a_{0} \cdots a_{p}} \int d^{p+1} \sigma H_{a_{0} \cdots a_{p-1}}\left(D^{d} \partial_{c} A_{b} D_{d}\left[\partial_{a_{p}} D^{c} \Phi_{i} D^{b} \Phi^{i}\right]-2 \leftrightarrow 3\right)
$$

To find all higher orders in $\alpha^{\prime}$ one must take into account the derivatives as appeared in (6.6) such that the final form is

$$
\begin{aligned}
S_{19}= & \frac{\left(2 \pi \alpha^{\prime}\right)^{3} \mu_{p} \epsilon^{a_{0} \cdots a_{p}} H_{a_{0} \cdots a_{p-1}}}{p !} \int d^{p+1} \sigma \times \\
& \times \sum_{p, n, m=0}^{\infty} f_{p, n, m}\left(\frac{\alpha^{\prime}}{2}\right)^{p}\left(\alpha^{\prime}\right)^{2 m+n}\left(D^{a} D_{a}\right)^{p}\left(D_{b_{1}} \cdots D_{b_{2 m}}\right. \\
& \left.\times D_{a_{1}} \cdots D_{a_{n}} \partial_{c} A_{b} D^{a_{1}} \cdots D^{a_{n}}\left[\partial_{a_{p}} D^{b_{1}} \cdots D^{b_{m}} D^{c} \Phi_{i} D^{b} D^{b_{m+1}} \cdots D^{b_{2 m}} \Phi^{i}\right]-2 \leftrightarrow 3\right)
\end{aligned}
$$

Of course for our amplitude $(C A \phi \phi)$ we just could confirm the presence of partial derivatives in the definitions of the covariant derivatives in the coupling (6.8) and it remains an open question to check whether or not the commutator in the definitions of covariant derivative of scalar fields will be kept. In order to answer this subtlety one must perform higher point functions, namely to compute either $C A A \phi \phi$ or $C A A A \phi \phi$. However there are 
some subtleties to answer this question and some of them have been addressed in $[15,31]$. We hope to answer some of them in future [57].

Note that these couplings are consistent with string amplitude of one $(p-1)$-form closed string RR and two scalar and one gauge field and they are new in the sense that neither do they come from Myers' terms, Pull-back, Taylor expansion nor expanding the exponential and suing the multiplication rule of the super matrices.

As it stands and it is known, applying the direct computations of string amplitudes is the only consistent and a quite reasonable method to actually find out new couplings in field theory.

Thus to conclude, in order to produce exactly the contact interactions in (6.1) at leading order one has to consider the sum of $S_{12}, S_{14}, S_{16}, S_{18}$ and consider these interactions by replacing $2 \leftrightarrow 3$ as well.

Finally we get to those contact terms that have been overlooked in section 4. By making use of some valuable formula we might write those interactions down as

$$
\begin{aligned}
& \frac{16 \pi \mu_{p}}{p !} \epsilon^{a_{0} \cdots a_{p-1} a} H_{a_{0} \cdots a_{p-1}} \xi_{3} \cdot \xi_{2}\left[\xi_{1 a} t s-2 t k_{3} \cdot \xi_{1} k_{2 a}-2 s k_{2} \cdot \xi_{1} k_{3 a}\right] \times \\
& \times \sum_{n, m=0}^{\infty}\left(a_{n, m}+b_{n, m}\right)\left(-\alpha^{\prime} k^{2}\right)^{l-1} \\
& \quad\left[\left(\begin{array}{c}
\left.2 \sum_{l=1}^{m}\left(\begin{array}{c}
m \\
l
\end{array}\right)\left(s^{m-l} t^{n}+t^{m-l} s^{n}\right)+2 \sum_{l=1}^{n}\left(\begin{array}{c}
n \\
l
\end{array}\right)\left(s^{n-l} t^{m}+t^{n-l} s^{m}\right)\right) \\
\left.+\sum_{l=1, j=1}^{n, m}\left(\begin{array}{c}
n \\
l
\end{array}\right)\left(\begin{array}{c}
m \\
j
\end{array}\right)\left(s^{n-l} t^{m-j}+t^{n-l} s^{m-j}\right)\left(-\alpha^{\prime} k^{2}\right)^{j}\right] \operatorname{Tr}\left(\lambda_{1} \lambda_{2} \lambda_{3}\right)
\end{array}\right.\right.
\end{aligned}
$$

The important point is that we can also write these contact terms in a closed form as follows

$$
\begin{aligned}
& \frac{16 \pi \mu_{p}}{p !} \epsilon^{a_{0} \cdots a_{p-1} a} H_{a_{0} \cdots a_{p-1}} \xi_{3} \cdot \xi_{2}\left[\xi_{1 a} t s-2 t k_{3} \cdot \xi_{1} k_{2 a}-2 s k_{2} \cdot \xi_{1} k_{3 a}\right] \\
& \times \operatorname{Tr}\left(\lambda_{1} \lambda_{2} \lambda_{3}\right) \sum_{p, n, m=0}^{\infty} f_{p, n, m}^{\prime}(s+t+u)^{p}(s+t)^{n}(s t)^{m}
\end{aligned}
$$

We should have pointed out the fact that $f_{p, n, m}^{\prime}$ can be written in terms of $a_{n, m}$ and $b_{n, m}$ as well. The last remark is that, the last terms in the expansion of $L_{1}^{\prime}$ do follow the same structures of (6.12). Therefore we can conclude that $f_{p, n, m}$ just in the expansion of $L_{1}^{\prime}$ must be replaced by

$$
f_{p, n, m} \rightarrow f_{p, n, m}-f_{p, n, m}^{\prime}
$$

\section{Contact terms for $p+2=n$ case}

Let us come to the last part of contact terms. Here all world volume spaces have been covered and apparently there should not be any coupling between gauge field and scalars and Ramond-Ramond. Below one might wonder how we could find a non-zero coupling 
between one closed string Ramond-Ramond $(p+1)$-form and a gauge field and two scalar fields in the world volume of BPS branes. In order to indeed explore new couplings, first we extract all traces and write down the general form of the non-zero amplitude(which is $A_{2}$ ), then we comment on new couplings which can be discovered just by direct super string scattering amplitudes in IIB and IIA theories.

$$
\begin{array}{r}
\mathcal{A}_{2}=L_{2} \pi^{1 / 2} \mu_{p} \frac{16}{(p+1) !} \xi_{2 i} \xi_{3 j}\left\{\epsilon ^ { a _ { 0 } \cdots a _ { p - 1 } c } H _ { a _ { 0 } \cdots a _ { p - 1 } } ^ { i j } ( p + 1 ) \left(-2 u s \xi_{1} \cdot k_{2} k_{3 c}+u s t \xi_{1 c}\right.\right. \\
\left.\left.\quad-2 u t k_{3} \cdot \xi_{1} k_{2 c}\right)+\epsilon^{a_{0} \cdots a_{p}}\left(2 u s p^{j} \xi_{1} \cdot k_{2} H_{a_{0} \cdots a_{p}}^{i}-2 u t p^{i} \xi_{1} \cdot k_{3} H_{a_{0} \cdots a_{p}}^{j}\right)\right\}
\end{array}
$$

It is extremely important to notice that, by applying momentum conservation all the terms in the first part of (7.1) do satisfy the only related Ward identity for the gauge field $\left(\xi_{1 c} \rightarrow k_{1 c}\right)$. However in order to see the fact that the same result holds for the other terms, we should apply the Bianchi identity as the following:

$$
\epsilon^{a_{0} \cdots a_{p}}\left(-p_{a_{p}}(p+1) H_{a_{0} \cdots a_{p-1}}^{i j}-p^{j} H_{a_{0} \cdots a_{p}}^{i}+p^{i} H_{a_{0} \cdots a_{p}}^{j}\right)=d H^{p+2}=0
$$

Regarding above results and in order to have gauge invariance at leading order of $\alpha^{\prime}$, we have to find out non-zero couplings for each term in the first part of (7.1) and then add them up. This rule should be kept for the last two terms in (7.1) as well. One may expand $u s t L_{2}^{\prime}$ as below

$$
t s u L_{2}^{\prime}=\pi^{3 / 2}\left(\sum_{n=-1}^{\infty} b_{n}(s+t)^{n+1}+\sum_{p, n, m=0}^{\infty} e_{p, n, m} u^{p+1}(t s)^{n}(t+s)^{m}\right)
$$

However, this is not the correct expansion here. Regarding the fact that all Gamma functions are symmetric under interchange of $(s, t, u)$, the final expansion must hold that symmetry as well so the modified expansion has to be taken as

$$
\begin{array}{r}
t s u L_{2}^{\prime}=\frac{\pi^{3 / 2}}{3}\left\{\left[\sum_{n=-1}^{\infty} b_{n}(s+t)^{n+1}+\sum_{p, n, m=0}^{\infty} e_{p, n, m} u^{p+1}(t s)^{n}(t+s)^{m}\right]\right. \\
+t \leftrightarrow u+s \leftrightarrow u\}
\end{array}
$$

Remember $e_{0,0,0}=0, b_{-1}=1$. First let us try to produce at leading order of $\alpha^{\prime}$ the second term of the string amplitude in (7.1), that is

$$
\pi^{2} \mu_{p} \frac{16}{p !} \xi_{2 i} \xi_{3 j} \epsilon^{a_{0} \cdots a_{p-1} c} H_{a_{0} \cdots a_{p-1}}^{i j} \xi_{1 c}
$$

Note that if we expand ust $L_{2}^{\prime}$ at low energy limit the first term is $\pi^{3 / 2}$.

One should argue that the coupling $\left(H^{p+2} A \phi \phi\right)$ has to be derived by field theory manipulation, basically consider the fact that both scalar fields could come from either Myers' terms as followed from (4.22) or both of them can be resulted in two covariant derivatives in the Pull-back like

$$
\frac{\left(2 \pi \alpha^{\prime}\right)^{2} \mu_{p}}{2(p-1) !} \int d^{p+1} \sigma \epsilon^{a_{0} \cdots a_{p}} \operatorname{Tr}\left(D_{a_{0}} \Phi^{i} D_{a_{1}} \Phi^{j}\right) C_{i j a_{2} \cdots a_{p}}
$$


The other possibility is that one scalar can come from pull-back and the second one could come from Taylor expansion as follows

$$
\frac{\left(2 \pi \alpha^{\prime}\right)^{2} \mu_{p}}{p !} \int d^{p+1} \sigma \epsilon^{a_{0} \cdots a_{p}} \operatorname{Tr}\left(\Phi^{j} D_{a_{0}} \Phi^{i}\right) \partial_{j} C_{i a_{1} \cdots a_{p}}
$$

As we can clearly see the presence of scalars (which are non-Abelian) in Taylor expansion [58-60] and pull-back should be inevitable as was mentioned in [61,62] and after all we have to extract covariant derivative of scalar to actually receive gauge, scalar and RR couplings. Needless to say we took integrations by parts to indeed combine (7.6) and (7.5), such that their combination is

$$
\frac{\left(2 \pi \alpha^{\prime}\right)^{2}}{2 p !} \mu_{p} \int d^{p+1} \sigma \epsilon^{a_{0} \cdots a_{p}} \operatorname{Tr}\left(D_{a_{0}} \Phi^{j} \Phi^{i}\right) H_{i j a_{1} \cdots a_{p}}
$$

If we would open up the covariant derivative of scalar field, we would get two terms but the term involving partial derivative should be dropped as we need to get the non-zero coupling of one gauge, two scalars and one $\mathrm{RR}-(p+1)$ form so that the ultimate coupling is achieved by

$$
\frac{i\left(2 \pi \alpha^{\prime}\right)^{2}}{p !} \mu_{p} \int d^{p+1} \sigma \epsilon^{a_{0} \cdots a_{p}} \operatorname{Tr}\left(A_{a_{0}} \Phi^{j} \Phi^{i}\right) H_{i j a_{1} \cdots a_{p}}
$$

It is indeed a very easy task to observe that (7.4) is precisely reproduced by (7.7). Again we want to highlight the point that even we are dealing with $n=p+2$ case and all world volume spaces have been covered, however, there is a non-zero coupling between $\operatorname{RR}-(p+1)$ form and one gauge field and two scalars field but we can no longer write that coupling in terms of field strength of the gauge field.

The higher derivative corrections of (7.7) can be discovered as

$$
\begin{aligned}
& \frac{i\left(2 \pi \alpha^{\prime}\right)^{2}}{3 p !} \mu_{p} \epsilon^{a_{0} \cdots a_{p}} H_{i j a_{1} \cdots a_{p}} \times \\
& \times\left(\int d ^ { p + 1 } \sigma \left[\sum_{n=-1}^{\infty} b_{n}\left(\alpha^{\prime}\right)^{n+1} \operatorname{Tr}\left(\partial_{m_{0}} \cdots \partial_{m_{n}} A_{a_{0}} D^{m_{0}} \cdots D^{m_{n}}\left[\Phi^{j} \Phi^{i}\right]\right)\right.\right. \\
& \quad+\sum_{p, n, m=0}^{\infty} e_{p, n, m}\left(\frac{\alpha^{\prime}}{2}\right)^{p+1}\left(\alpha^{\prime}\right)^{2 n+m} \operatorname{Tr}\left(\partial_{m_{1}} \cdots \partial_{m_{m}} \partial_{n_{1}} \cdots \partial_{n_{2 n}} A_{a_{0}} \partial^{m_{1}} \cdots \partial^{m_{m}}\right. \\
& \left.\left.\left.\times\left(D_{c} D^{c}\right)^{p+1}\left[D^{n_{1}} \cdots D^{n_{n}} \Phi^{j} D^{n_{n+1}} \cdots D^{n_{2 n}} \Phi^{i}\right]\right)\right]\right)
\end{aligned}
$$

This prescription can be easily applied to actually get the terms by interchanging $t \leftrightarrow u, s \leftrightarrow u$ and finally we have to add them to (7.8) as well. Now let us consider the first and third terms in (7.1) and just keep the related leading contact interactions, namely one must employ $u s L_{2}^{\prime}, u t L_{2}^{\prime}$ expansions in (2.21) and (2.22) and keep in mind that the first non zero coefficients are $e_{1,0,0}, e_{0,0,1}$ such that the following terms are leading terms in string amplitude

$$
-\pi^{4} \mu_{p} \frac{16}{6 p !} \xi_{2 i} \xi_{3 j} \epsilon^{a_{0} \cdots a_{p-1} c} H_{a_{0} \cdots a_{p-1}}^{i j}\left(-2 \xi_{1} \cdot k_{2} k_{3 c}(t+2 s+2 u)-2 k_{3} \cdot \xi_{1} k_{2 c}(s+2 t+2 u)\right)
$$


In order to produce all terms in (7.9), one has to work in detail and write down some new couplings, in the sense that they do not come from pull-back, Taylor or Myers' terms. Therefore the first term above can be reproduced by the following coupling:

$$
\begin{aligned}
-\frac{4 \pi^{2}\left(2 \pi \alpha^{\prime}\right)^{2}}{6 p !} \mu_{p} \int d^{p+1} \sigma & \left(\partial_{b} A_{a} D^{b} D^{a} \Phi^{i} D_{c} \Phi^{j}+2 \partial_{b} A_{a} D^{a} \Phi^{i} D_{c} \partial^{b} \Phi^{j}\right. \\
+ & \left.2 A_{a} D^{a} \partial_{b} \Phi^{i} D_{c} \partial^{b} \Phi^{j}-2 \leftrightarrow 3\right) H_{i j a_{0} \cdots a_{p-1}}^{(p+2)} \epsilon^{a_{0} \cdots a_{p-1} c}
\end{aligned}
$$

Now we get to the point which has been emphasized, namely in order to produce all the first three terms in (7.1), one has to add (7.7) and (7.10) together, and generalizing to all orders can be easily done with making use of (6.1).

Now let us consider the last two terms in (7.1) and just keep the related leading contact interactions. Having set the $u s L_{2}^{\prime}, u t L_{2}^{\prime}$ expansions from (2.21) and (2.22) we get

$$
-\pi^{4} \mu_{p} \frac{16}{6(p+1) !} \xi_{2 i} \xi_{3 j} \epsilon^{a_{0} \cdots a_{p}}\left(2 p^{j} \xi_{1} \cdot k_{2} H_{a_{0} \cdots a_{p}}^{i}(t+2 s+2 u)-2 p^{i} k_{3} \cdot \xi_{1} H_{a_{0} \cdots a_{p}}^{j}(s+2 t+2 u)\right)
$$

By applying the correct higher derivative corrections, it is easy to show that some new couplings must be taken in to account, such that the first term above can be reproduced by the following coupling:

$$
\begin{aligned}
\frac{4 \pi^{2}\left(2 \pi \alpha^{\prime}\right)^{2}}{6(p+1) !} \mu_{p} \int d^{p+1} \sigma\left(\partial_{b} A_{a} D^{b} D^{a} \Phi^{i} \Phi^{j}+2 \partial_{b} A_{a} D^{a} \Phi^{i} D^{b} \Phi^{j}\right. & \\
& \left.+2 A_{a} D^{b} D^{a} \Phi^{i} D_{b} \Phi^{j}-2 \leftrightarrow 3\right) \partial_{j} H_{a_{0} \cdots a_{p}}^{i} \epsilon^{a_{0} \cdots a_{p}}
\end{aligned}
$$

Again we want to highlight the point that these new couplings do not come from pullback or Taylor or Myers' terms. Having used (6.1), we can easily generalize above couplings to actually get all contact interactions to all orders of $\alpha^{\prime}$.

However, it turns out that it is better to write down the closed form of contact terms to all orders of $\alpha^{\prime}$ rather than producing them order by order in $\alpha^{\prime}$. Thus consider the following terms in string amplitude

$$
-\pi^{2} \mu_{p} \frac{16}{p !} \xi_{2 i} \xi_{3 j} \epsilon^{a_{0} \cdots a_{p-1} c} H_{a_{0} \cdots a_{p-1}}^{i j} \sum_{p, n, m=0}^{\infty} e_{p, n, m}\left(-2 \xi_{1} \cdot k_{2} k_{3 c} t^{p}(s u)^{n}(s+u)^{m}-2 \leftrightarrow 3\right)
$$

Their closed form can be precisely obtained to all orders of $\alpha^{\prime}$ by the following coupling:

$$
\begin{gathered}
\frac{\mu_{p}\left(2 \pi \alpha^{\prime}\right)^{2}}{p !} \sum_{p, n, m=0}^{\infty} e_{p, n, m}\left(\frac{\alpha^{\prime}}{2}\right)^{p}\left(\alpha^{\prime}\right)^{2 n+m+1} \int d^{p+1} \sigma\left(( D _ { a } D ^ { a } ) ^ { p } D _ { a _ { 1 } } \cdots D _ { a _ { m } } \left[\partial_{a_{1}} \cdots \partial_{a_{n}} A_{a}\right.\right. \\
\left.\left.\times D_{a_{n+1}} \cdots D_{a_{2 n}} D^{a} \Phi^{i}\right] D_{a_{p}} D^{a_{1}} \cdots D^{a_{m}} D^{a_{1}} \cdots D^{a_{2 n}} \Phi^{j}+2 \leftrightarrow 3\right) H_{a_{0} \cdots a_{p-1}}^{i j} \epsilon^{a_{0} \cdots a_{p}}
\end{gathered}
$$

Finally the rest of the terms in string amplitude to all orders are verified as

$$
-\pi^{2} \mu_{p} \frac{16}{(p+1) !} \xi_{2 i} \xi_{3 j} \epsilon^{a_{0} \cdots a_{p}} \sum_{p, n, m=0}^{\infty} e_{p, n, m} t^{p}(s u)^{n}(s+u)^{m}\left(2 p^{j} \xi_{1} \cdot k_{2} H_{a_{0} \cdots a_{p}}^{i}-2 \leftrightarrow 3\right)
$$


Eventually by applying the same methodology as discussed in the body of the paper, one can easily show that (7.15) would be produced by the following couplings:

$$
\begin{array}{r}
\frac{\mu_{p}\left(2 \pi \alpha^{\prime}\right)^{2}}{(p+1) !} \sum_{p, n, m=0}^{\infty} e_{p, n, m}\left(\frac{\alpha^{\prime}}{2}\right)^{p}\left(\alpha^{\prime}\right)^{2 n+m+1} \int d^{p+1} \sigma\left(( D _ { a } D ^ { a } ) ^ { p } D _ { a _ { 1 } } \cdots D _ { a _ { m } } \left[\partial_{a_{1}} \cdots \partial_{a_{n}} A_{a}\right.\right. \\
\left.\left.\times D_{a_{n+1}} \cdots D_{a_{2 n}} D^{a} \Phi^{i}\right] D^{a_{1}} \cdots D^{a_{m}} D^{a_{1}} \cdots D^{a_{2 n}} \Phi^{j}-2 \leftrightarrow 3\right) \partial_{j} H_{i a_{0} \cdots a_{p}} \epsilon^{a_{0} \cdots a_{p}}
\end{array}
$$

\section{Conclusion}

First of all by applying conformal field theory methods we discovered the complete result of the amplitude of one Ramond-Ramond, one gauge field and two scalar fields for all kinds of $p$ and $n$ in II string theory. The motivation for carrying out this long computation was that, we must have the complete form of the amplitude to be able to proceed to explore the closed form of new Wess-Zumino couplings to all orders in $\alpha^{\prime}$ for various cases. We have also performed all SYM vertex operators to all orders in $\alpha^{\prime}$. Remember that due to closed string Ramond-Ramond the general form of these new couplings with their exact coefficients should be confirmed just by direct S-Matrix computations not any other tool like T-duality transformation to the previous calculations. The results of this paper can not be found for example from $<V_{C} V_{A} V_{A} V_{\phi}>$ because of the fact that $C$-vertex operator does not have Winding modes in its form, which means that all the terms including $p^{i}, p^{j}$ of this paper have not been showed up in $\left\langle V_{C} V_{A} V_{A} V_{\phi}>\right.$. We have shown that the amplitude of $C A \phi \phi$ has infinite massless poles in various channels. Namely making use of the all order two gauge two scalar couplings [29], we were able to match all infinite massless gauge poles in $(t+s+u)$-channel in string theory amplitude with field theory computations. We have also produced all infinite massless scalar poles in t,s-channels in both field and string theory sides. Apart from those things, we showed that the amplitude has again infinite massless gauge poles in u-channel for $p=n+2$ case and then we went through new WZ couplings for this case like $\operatorname{Tr}\left(C_{p-3} \wedge F \wedge D \phi^{i} \wedge D \phi_{i}\right)$ and all its infinite higher derivative corrections have been explored in (4.1).

It has been eventually clarified that the couplings of two scalars and one gauge field can not hold any corrections, thus all non-leading (gauge/scalar) poles have provided the most needed information to indeed get the all order $\alpha^{\prime}$ higher derivative corrections to $\operatorname{Tr}\left(C_{p-3} \wedge F \wedge F\right)$ and $\left(\operatorname{Tr}\left(\phi^{j} \phi^{i}\right) \partial_{j} H_{i a_{0} \cdots a_{p}}+(p+1) \operatorname{Tr}\left(\partial_{a_{0}} \phi^{j} \phi^{i}\right) H_{i j a_{1} \cdots a_{p}}\right)$.

We also found further results, basically in order to produce all infinite $t, s$-channel scalar poles we found (4.28). To get all infinite contact interactions for $p=n$ case, (5.2), (6.1) are derived. New couplings and all their infinite $\alpha^{\prime}$ corrections are discovered in (6.4)-(7.1). In order to actually derive all contact terms for $p+2=n$ case, we have also obtained several new Wess-Zumino couplings in (7.15), (7.13) with all their infinite corrections in (7.15) and (7.16) and also (7.9) is derived to all orders. These new interactions which are neither inside Myers'terms nor within pull-back/Taylor expansion must be looked for only by performing direct string computations. It would be nice to perform either $C A A \phi \phi$ or 
$C A A A \phi \phi$ to get some more information, remove some of the ambiguities which are addressed in this paper and finally to see whether or not covariant derivatives should be kept inside the new couplings of this paper.

\section{Acknowledgments}

It is my pleasure to thank Rob Myers for several useful discussions and for sharing his ideas on some new WZ couplings with me. The author would like to thank J.Polchinski, K.S.Narain, E.Gava and F.Quevedo for valuable discussions. He acknowledges E.Witten, G.W.Moore, W.Taylor and J.Maldacena for useful discussions during Strings 2012 held at Munich also thanks L. Álvarez-Gaumé, N.Lambert and G.Veneziano for several valuable comments. He also thanks Perimeter Institute for warm hospitality during his visit where some part of this work was taken place there.

Open Access. This article is distributed under the terms of the Creative Commons Attribution License which permits any use, distribution and reproduction in any medium, provided the original author(s) and source are credited.

\section{References}

[1] J. Polchinski, Dirichlet branes and Ramond-Ramond charges, Phys. Rev. Lett. 75 (1995) 4724 [hep-th/9510017] [INSPIRE].

[2] E. Witten, Bound states of strings and p-branes, Nucl. Phys. B 460 (1996) 335 [hep-th/9510135] [INSPIRE].

[3] J. Polchinski, TASI lectures on D-branes, hep-th/9611050 [INSPIRE].

[4] C.P. Bachas, Lectures on D-branes, hep-th/9806199 [INSPIRE].

[5] M. Li, Boundary states of D-branes and Dy strings, Nucl. Phys. B 460 (1996) 351 [hep-th/9510161] [INSPIRE].

[6] M.B. Green, J.A. Harvey and G.W. Moore, I-brane inflow and anomalous couplings on D-branes, Class. Quant. Grav. 14 (1997) 47 [hep-th/9605033] [INSPIRE].

[7] E. Witten, Small instantons in string theory, Nucl. Phys. B 460 (1996) 541 [hep-th/9511030] [INSPIRE].

[8] M.R. Douglas, Branes within branes, hep-th/9512077 [INSPIRE].

[9] R. Minasian and G.W. Moore, K theory and Ramond-Ramond charge, JHEP 11 (1997) 002 [hep-th/9710230] [INSPIRE].

[10] E. Witten, D-branes and k-theory, JHEP 12 (1998) 019 [hep-th/9810188] [INSPIRE].

[11] R.C. Myers, Dielectric branes, JHEP 12 (1999) 022 [hep-th/9910053] [INSPIRE].

[12] W. Taylor and M. Van Raamsdonk, Multiple D0-branes in weakly curved backgrounds, Nucl. Phys. B 558 (1999) 63 [hep-th/9904095] [INSPIRE].

[13] W. Taylor and M. Van Raamsdonk, Multiple Dp-branes in weak background fields, Nucl. Phys. B 573 (2000) 703 [hep-th/9910052] [INSPIRE]. 
[14] J. Polchinski, String duality: a colloquium, Rev. Mod. Phys. 68 (1996) 1245 [hep-th/9607050] [INSPIRE].

[15] E. Hatefi, On effective actions of BPS branes and their higher derivative corrections, JHEP 05 (2010) 080 [arXiv:1003.0314] [INSPIRE].

[16] A.A. Tseytlin, Born-Infeld action, supersymmetry and string theory, hep-th/9908105 [INSPIRE].

[17] A.A. Tseytlin, On non-abelian generalization of Born-Infeld action in string theory, Nucl. Phys. B 501 (1997) 41 [hep-th/9701125] [INSPIRE].

[18] R. Leigh, Dirac-Born-Infeld action from Dirichlet $\sigma$-model, Mod. Phys. Lett. A 4 (1989) 2767 [INSPIRE].

[19] P. Howe, U. Lindström and L. Wulff, On the covariance of the Dirac-Born-Infeld-Myers action, JHEP 02 (2007) 070 [hep-th/0607156] [INSPIRE].

[20] M. Cederwall, A. von Gussich, B.E. Nilsson and A. Westerberg, The Dirichlet super three-brane in ten-dimensional type IIB supergravity, Nucl. Phys. B 490 (1997) 163 [hep-th/9610148] [inSPIRE].

[21] M. Aganagic, C. Popescu and J.H. Schwarz, D-brane actions with local kappa symmetry, Phys. Lett. B 393 (1997) 311 [hep-th/9610249] [INSPIRE].

[22] M. Aganagic, C. Popescu and J.H. Schwarz, Gauge invariant and gauge fixed D-brane actions, Nucl. Phys. B 495 (1997) 99 [hep-th/9612080] [INSPIRE].

[23] M. Cederwall, A. von Gussich, B.E. Nilsson, P. Sundell and A. Westerberg, The Dirichlet super p-branes in ten-dimensional type IIA and IIB supergravity, Nucl. Phys. B 490 (1997) 179 [hep-th/9611159] [inSPIRE].

[24] E. Bergshoeff and P. Townsend, Super D-branes, Nucl. Phys. B 490 (1997) 145 [hep-th/9611173] [INSPIRE].

[25] E. Hatefi, Closed string Ramond-Ramond proposed higher derivative interactions on fermionic amplitudes in IIB, arXiv:1302.5024 [INSPIRE].

[26] A. Bilal, Higher derivative corrections to the non-abelian Born-Infeld action, Nucl. Phys. B 618 (2001) 21 [hep-th/0106062] [INSPIRE].

[27] O. Chandía and R. Medina, Four point effective actions in open and closed superstring theory, JHEP 11 (2003) 003 [hep-th/0310015] [INSPIRE].

[28] L.A. Barreiro and R. Medina, 5-field terms in the open superstring effective action, JHEP 03 (2005) 055 [hep-th/0503182] [INSPIRE].

[29] E. Hatefi and I. Park, More on closed string induced higher derivative interactions on D-branes, Phys. Rev. D 85 (2012) 125039 [arXiv:1203.5553] [INSPIRE].

[30] E. Hatefi and I. Park, Universality in all-order $\alpha^{\prime}$ corrections to BPS/non-BPS brane world volume theories, Nucl. Phys. B 864 (2012) 640 [arXiv: 1205.5079] [INSPIRE].

[31] E. Hatefi, On higher derivative corrections to Wess-Zumino and tachyonic actions in type-II super string theory, Phys. Rev. D 86 (2012) 046003 [arXiv:1203.1329] [INSPIRE].

[32] M.R. Garousi and E. Hatefi, More on WZ action of non-BPS branes, JHEP 03 (2009) 008 [arXiv:0812.4216] [INSPIRE]. 
[33] M.R. Garousi and E. Hatefi, On Wess-Zumino terms of brane-antibrane systems, Nucl. Phys. B 800 (2008) 502 [arXiv:0710.5875] [InSPIRE].

[34] E. Hatefi, On D-brane anti D-brane effective actions and their corrections to all orders in alpha-prime, arXiv:1211.5538 [INSPIRE].

[35] E. Hatefi, A. Nurmagambetov and I. Park, ADM reduction of IIB on $H^{p, q}$ to dS braneworld, arXiv:1210.3825 [INSPIRE].

[36] I. Park, Geometric counter-vertex for open string scattering on D-branes, Eur. Phys. J. C 67 (2010) 543 [arXiv:0902.1279] [inSPIRE].

[37] P. Koerber and A. Sevrin, The non-abelian D-brane effective action through order $\alpha^{4}$, JHEP 10 (2002) 046 [hep-th/0208044] [INSPIRE].

[38] A. Keurentjes, P. Koerber, S. Nevens, A. Sevrin and A. Wijns, Towards an effective action for D-branes, Fortsch. Phys. 53 (2005) 599 [hep-th/0412271] [INSPIRE].

[39] F. Denef, A. Sevrin and J. Troost, Non-abelian Born-Infeld versus string theory, Nucl. Phys. B 581 (2000) 135 [hep-th/0002180] [INSPIRE].

[40] E. Hatefi, A. Nurmagambetov and I. Park, $N^{3}$ entropy of $M 5$ branes from dielectric effect, Nucl. Phys. B 866 (2013) 58 [arXiv:1204.2711] [INSPIRE].

[41] E. Hatefi, A. Nurmagambetov and I. Park, Near-extremal black-branes with $n^{3}$ entropy growth, Int. J. Mod. Phys. A 27 (2012) 1250182 [arXiv:1204.6303] [INSPIRE].

[42] J. McOrist and S. Sethi, M-theory and type IIA flux compactifications, JHEP 12 (2012) 122 [arXiv: 1208.0261] [INSPIRE].

[43] A. Fotopoulos, On $\left(\alpha^{\prime}\right)^{2}$ corrections to the D-brane action for nongeodesic world volume embeddings, JHEP 09 (2001) 005 [hep-th/0104146] [INSPIRE].

[44] H. Liu and J. Michelson, *-trek 3: the search for Ramond-Ramond couplings, Nucl. Phys. B 614 (2001) 330 [hep-th/0107172] [INSPIRE].

[45] S. Stieberger, Open $\& 3$ closed vs. pure open string disk amplitudes, arXiv:0907.2211 [INSPIRE].

[46] J. Polchinski and M.J. Strassler, The string dual of a confining four-dimensional gauge theory, hep-th/0003136 [INSPIRE].

[47] K. Pilch and N.P. Warner, $N=1$ supersymmetric renormalization group flows from IIB supergravity, Adv. Theor. Math. Phys. 4 (2002) 627 [hep-th/0006066] [INSPIRE].

[48] O. Aharony and A. Rajaraman, String theory duals for mass deformed $\mathrm{SO}(N)$ and $\mathrm{USp}(2 N)$ $N=4$ SYM theories, Phys. Rev. D 62 (2000) 106002 [hep-th/0004151] [INSPIRE].

[49] I. Bena, The M-theory dual of a three-dimensional theory with reduced supersymmetry, Phys. Rev. D 62 (2000) 126006 [hep-th/0004142] [INSPIRE].

[50] I. Bena and A. Nudelman, Warping and vacua of $(S) Y M(2+1)$, Phys. Rev. D 62 (2000) 086008 [hep-th/0005163] [INSPIRE].

[51] C. Bachas, M.R. Douglas and C. Schweigert, Flux stabilization of D-branes, JHEP 05 (2000) 048 [hep-th/0003037] [INSPIRE].

[52] M. Li, Fuzzy gravitons from uncertain space-time, Phys. Rev. D 63 (2001) 086002 [hep-th/0003173] [INSPIRE]. 
[53] M.T. Grisaru, R.C. Myers and O. Tafjord, SUSY and Goliath, JHEP 08 (2000) 040 [hep-th/0008015] [INSPIRE].

[54] S.P. Trivedi and S. Vaidya, Fuzzy cosets and their gravity duals, JHEP 09 (2000) 041 [hep-th/0007011] [INSPIRE].

[55] M.R. Garousi and R.C. Myers, World volume potentials on D-branes, JHEP 11 (2000) 032 [hep-th/0010122] [INSPIRE].

[56] L.A. Barreiro and R. Medina, Revisiting the S-matrix approach to the open superstring low energy effective lagrangian, JHEP 10 (2012) 108 [arXiv:1208.6066] [INSPIRE].

[57] E.Hatefi, Answering fundamental questions in super string theory, work in progress.

[58] M.R. Douglas, A. Kato and H. Ooguri, D-brane actions on Kähler manifolds, Adv. Theor. Math. Phys. 1 (1998) 237 [hep-th/9708012] [INSPIRE].

[59] M.R. Douglas, D-branes and matrix theory in curved space, Nucl. Phys. Proc. Suppl. 68 (1998) 381 [hep-th/9707228] [InSPIRE].

[60] E. Hatefi, Three point tree level amplitude in superstring theory, Nucl. Phys. Proc. Suppl. 216 (2011) 234 [arXiv:1102.5042] [InSPIRE].

[61] H. Dorn, NonAbelian gauge field dynamics on matrix D-branes, Nucl. Phys. B 494 (1997) 105 [hep-th/9612120] [INSPIRE].

[62] C. Hull, Matrix theory, $U$ duality and toroidal compactifications of $M$-theory, JHEP 10 (1998) 011 [hep-th/9711179] [INSPIRE]. 